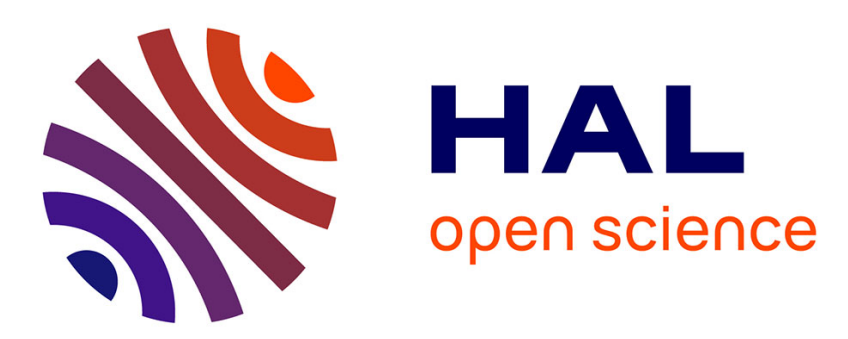

\title{
General relations to obtain the time-dependent heat capacity from isothermal simulations
}

L. Klochko, J. Baschnagel, J. P Wittmer, Alexander N Semenov

\section{To cite this version:}

L. Klochko, J. Baschnagel, J. P Wittmer, Alexander N Semenov. General relations to obtain the time-dependent heat capacity from isothermal simulations. Journal of Chemical Physics, 2021, 154 (16), pp.164501. 10.1063/5.0046697 . hal-03255166

\section{HAL Id: hal-03255166 https://hal.science/hal-03255166}

Submitted on 9 Jun 2021

HAL is a multi-disciplinary open access archive for the deposit and dissemination of scientific research documents, whether they are published or not. The documents may come from teaching and research institutions in France or abroad, or from public or private research centers.
L'archive ouverte pluridisciplinaire HAL, est destinée au dépôt et à la diffusion de documents scientifiques de niveau recherche, publiés ou non, émanant des établissements d'enseignement et de recherche français ou étrangers, des laboratoires publics ou privés. 


\section{General relations to obtain the time-dependent heat capacity from isothermal}

simulations

L.Klochko, J.Baschnagel, J.P.Wittmer, A.N.Semenov ${ }^{\text {a) } 1}$

Institut Charles Sadron, CNRS - UPR 22, Université de Strasbourg, 23 rue du Loess, BP 84047, 67034 Strasbourg Cedex 2, France

(Dated: 17 March 2021)

It is well-known that time-dependent correlation functions related to temperature and energy can crucially depend on the thermostatting mechanism used in computer simulations of molecular systems. We argue, however, that linear response functions must be considered as universal properties of physical systems. These features imply that the classical fluctuation equation for the transient heat capacity, $c_{v}(t)$, is not applicable to the thermostatted molecular dynamics (apart from long enough times). To improve on this point we derive a number of exact general expressions for the frequency-dependent heat capacity in terms of energy correlation functions, valid for the Nosé-Hoover and some other thermostats. We also establish a general relation between auto- and cross-correlation functions of energy and temperature. Recommendations on how to use these relations to maximize the numerical precision are provided. It is demonstrated that our approach allows to obtain $c_{v}(t)$ for a supercooled liquid system with high precision and over many decades in time reflecting all pertinent relaxation processes.

a) Author to whom correspondence should be addressed: al.ni.semenov@gmail.com 


\section{INTRODUCTION}

Molecular systems are ubiquitely characterized by their response to small external perturbations. The perturbations can range from a deformation (shear, extension or compression) to pressure or temperature variation (not to mention dielectric response). It is also well-known that, by virtue of the fluctuation-dissipation theorem $(\mathrm{FDT})^{1-4}$, such macroscopic response functions are related to correlation functions of the relevant physical variables measured for the unperturbed equilibrium system. For example, the shear-stress relaxation modulus $G(t)$ providing the shear stress increment upon a step-like shear deformation is related to the shear-stress autocorrelation function $^{5-9}$. The bulk relaxation modulus $K_{b}(t)$ defining pressure response to compression is proportional to the autocorrelation function of the normal pressure (with an additional constant) ${ }^{8,10}$. In the same spirit, the heat capacity $c_{v}$ is related to fluctuations of total energy (or entropy) for a system kept at a constant temperature $T$ and constant volume (in the canonical ensemble) ${ }^{1,2}$ :

$$
N c_{v s}=\frac{1}{T^{2}}\left\langle(E-\langle E\rangle)^{2}\right\rangle
$$

where $N$ is the number of particles in the system, $c_{v s}$ is the static heat capacity per particle, $E=E(t)$ is the total energy of the system at time $t$, and $\langle\ldots\rangle$ denote the canonical equilibrium average. ${ }^{57}$

In systems with long relaxation times (reluctantly changing their configurations) the heat capacity depends on time, $c_{v s} \rightarrow c_{v}(t)$. Notable examples include polymer melts and concentration solutions, supercooled liquids and all sorts of glasses ${ }^{2,11}$. The function $c_{v}(t)$ can be defined as the amount of heat, $q(t)$, absorbed during time $t$ in response to a small step-like increase of temperature, $\Delta T$, at $t=0: c_{v}(t)=q(t) / \Delta T, t>0$. Here $q$ is the heat per particle which under the assumed isochoric conditions must be related to the energy change: $q(t)=\langle E(t)-E(-\infty)\rangle / N$. The dynamic nature of $c_{v}$ can be alternatively expressed by its frequency dependence: $c_{v}^{*}(\omega)=q_{\omega} / T_{\omega}$, where $q_{\omega}$ and $T_{\omega}$ are the complex Fourier amplitudes of $q(t)$ and $\Delta T(t)=T(t)-T(-\infty)$, respectively (in the case of a harmonic oscillatory perturbation $\Delta T(t)=T_{\omega} e^{\mathrm{i} \omega t}$ and $q(t)=q_{\omega} e^{\mathrm{i} \omega t 58}$ ). The complex dynamic heat capacity $c_{v}^{*}(\omega)$ is related to $c_{v}(t)$ by:

$$
c_{v}^{*}(\omega)=c_{v}^{\prime}(\omega)+\mathrm{i} c_{v}^{\prime \prime}(\omega)=\mathrm{i} \omega \int_{0}^{\infty} c_{v}(t) e^{-\mathrm{i} \omega t} \mathrm{~d} t
$$

since

$$
q(t)=\int_{-\infty}^{t} c_{v}\left(t-t^{\prime}\right) \mathrm{d} T\left(t^{\prime}\right)=\int_{-\infty}^{t} c_{v}\left(t-t^{\prime}\right) \frac{\mathrm{d} T}{\mathrm{~d} t^{\prime}} \mathrm{d} t^{\prime}
$$


by virtue of the (Boltzmann) superposition principle of linear response theory. Note that eq. 2 follows from the definitions of $c_{v}(t)$ and $c_{v}^{*}(\omega)$ discussed above. The definition of $c_{v}^{*}(\omega)$ adopted here is equivalent to the standard definition of the dynamic heat capacity commonly used in the literature (cf. ref. ${ }^{56}$ ). Noteworthily, eq. 2 is analogous to the well-known relation between the complex dynamic modulus $G^{*}(\omega)=G^{\prime}(\omega)+\mathrm{i} G^{\prime \prime}(\omega)^{11}$ and the shear relaxation modulus $G(t)$.

The existence of a time-dependent specific heat in glasses and supercooled liquids near the glass transition had been predicted ${ }^{13-15}$ and observed experimentally ${ }^{15-19}$. The time-dependent specific heat was also considered theoretically within a generalized hydrodynamic approach (using MoriZwanzig formalism) $)^{3,12,20,21}$.

In both experimental and simulation studies it is normally preferable to deal with systems whose temperature is controlled by means of an appropriate thermostat. In this paper we discuss how to obtain $c_{v}(t)$ based on equilibrium dynamics of a model system studied by molecular dynamics (MD) simulations.

Below we first outline the well-known relation between $c_{v}(t)$ and equilibrium energy fluctuations in thermostatted systems. We then show that this relation is not applicable in the whole time-range (section II). In section III we introduce a physical Ansatz stating that response functions in general and $c_{v}(t)$ in particular must be universal (thermostat-independent). By contrast, the dynamic correlation functions depend on the thermostat properties as demonstrated in section II. This renders importance to the following question: Is it possible to get the time-dependent heat capacity $c_{v}(t)$ based on correlation functions in isothermal simulations? In this paper we show that while the answer is positive, it is not as trivial as it may seem to be. In sections III - VI we derive a number of general relations which are expected to universally define $c_{v}(t)$ and $c_{v}^{*}(\omega)$ in terms of equilibrium correlation functions and demonstrate their validity for the Nosé-Hoover thermostatting mechanism. The generalizations of these relations to non-canonical thermostats (like Gaussian isokinetic thermostat) are presented in section VII A. The region of applicability of the universality principle $\left(c_{v}(t)\right.$ independence of the thermostat parameters) is discussed in section VII B. The main results are summarized in the last section.

\section{THE FDT RELATION FOR THE HEAT CAPACITY}

While the total energy $E$ is conserved in microcanonical simulations, it is often desirable to impose a certain temperature $T=T_{\mathrm{i}}$ rather than energy. Such NVT simulations can be done 
using different mechanisms of temperature control ${ }^{10,22,23}$. A number of thermostatting methods ${ }^{24}$ have been proposed, including simulation techniques that sample a true canonical ensemble, in particular, the Langevin and Nosé-Hoover $(\mathrm{NH})$ canonical thermostats. ${ }^{25-27}$

For thermostatted systems the time-dependent heat capacity $c_{v 0}(t)$ was defined via the energy response to an infinitesimal step-like increase of the thermostat temperature $T_{\mathrm{i}}$ :

$$
T_{i} \rightarrow T_{i}+\varepsilon \text { at } t=0
$$

An exact expression for thus defined $c_{\nu 0}(t)=\langle E(t)-E(-\infty)\rangle /(N \varepsilon)$ (the additional subscript ' 0 ' emphasizes its relation to a thermostat) in terms of the energy correlation function was derived in ref. $^{28}$ based on the FDT $^{1-4}$ :

$$
c_{v 0}(t)=\frac{1}{T_{i}^{2}}\left[C_{E}(0)-C_{E}(t)\right]
$$

where $C_{E}(t)$ is the autocorrelation function of the total energy:

$$
C_{E}(t)=\frac{1}{N}\left\langle\delta E\left(t+t^{\prime}\right) \delta E\left(t^{\prime}\right)\right\rangle
$$

and $\delta E(t)=E(t)-\bar{E}$ is the energy fluctuation ( $\bar{E}$ is the time-averaged energy). Similar expressions for isobaric heat capacity $c_{p}(t)$ in terms of enthalpy $\left(H=E+p_{\mathrm{i}} V\right)$ fluctuations have been proposed long ago ${ }^{15,28}$ and have been used to interpret the results of dynamic heat spectroscopy (ac calorimetry) $)^{15,29,30}$ and multifrequency calorimetric data ${ }^{31}$.

We employed eq. 6 to calculate $c_{v 0}(t)$ for a two-dimensional (2D) polydisperse Lennard-Jones (pLJ) liquid described in Appendix A. The energy correlation function was obtained based on MD simulations with Nosé-Hoover (NH) thermostat as implemented in the LAMMPS code ${ }^{32}$. All quantities will be given in Lennard-Jones (LJ) units in what follows (see Appendix A). The results for $N=10^{4}$ particles at $T=0.4$ obtained using a single NH thermostat (LAMMPS parameter 'tchain $=1$ ') and different thermal mass $Q$ (cf. Appendix B), $Q=10 T / 3,100 T, N T / 10,2 N T$ (corresponding to the Nosé relaxation times ${ }^{33} \tau_{N}=\sqrt{2} / \omega_{Q}=0.013,0.071,0.22,1$, respectively, where $\omega_{Q}=(2 T N d / Q)^{1 / 2}$ is the Nosé frequency and the space dimension $\left.d=2\right)$ are shown in Fig. 1. It is obvious that $c_{v 0}(t)$ strongly depends on the thermostat-related parameter $Q$ and the results for all $4 Q$ 's agree only for $t>200$ where $c_{v 0}(t)$ appears to be nearly constant, $c_{v 0}(t) \approx c_{v s}$ $\left(c_{v s} \approx 1.947\right.$ is the static heat capacity per particle obtained using eq. 1 by averaging the energy fluctuations over the total sampling time $\Delta t=10^{5}$ of the production run, cf. Appendix A). ${ }^{59}$

Thus, it is obvious that while the relation, eq. 5, is applicable at long enough times, it dramatically fails at short times: both $c_{v 0}(t)$ and the energy correlation function $C_{E}(t)$ strongly depend on 
$Q$ (more generally, on the thermostat properties) in this regime.

This issue was recognized before ${ }^{26,33,34}$ as reflected in the following extract from ref. ${ }^{34}$. ' ... the problem is that there is no method by which to perform isothermal simulations without influencing the energy fluctuations of the system in an artificial way, which clearly spoils the dynamic response functions.' However, to the best of our knowledge, no satisfactory solution to this problem was provided for thermostatted systems.

Is it possible, based on the thermostatted dynamics, to get universal response functions (like the dynamic heat capacity) reflecting the dynamical properties of the original physical system? The main goal of the present paper is not only to demonstrate that the answer is positive, but also to provide straightforward practical recipes on how to calculate such universal responses.

\section{THE UNIVERSAL DYNAMIC HEAT CAPACITY}

To get the correct dynamic heat capacity $c_{v}^{*}(\omega)$ (and its time-dependent counterpart, $c_{v}(t)$ ) we must use its appropriate physical definition. Following refs. ${ }^{10,12,34}$ we define $c_{v}(t)$ via the energy response $\langle\Delta E(t)\rangle=\langle E(t)-E(-\infty)\rangle$ to a perturbation of the internal temperature of the system, $\langle\Delta T(t)\rangle=\langle T(t)-T(-\infty)\rangle$, where the instantaneous temperature $T(t)$ is related to the total kinetic energy $K(t)$ :

$$
T(t)=2 K(t) / g
$$

where $g$ is the number of kinetic degrees of freedom. ${ }^{60}$ An excellent discussion on why this 'kinetic' definition is appropriate is given in ref. ${ }^{12}$. Using $\langle\Delta T(t)\rangle=T_{\omega} e^{\mathrm{i} \omega t}$ and $\langle\Delta E(t)\rangle=E_{\omega} e^{\mathrm{i} \omega t}$ one gets

$$
c_{v}^{*}(\omega)=\frac{1}{N} \frac{E_{\omega}}{T_{\omega}}
$$

in the limit of an infinitesimal $T$-perturbation. The definition of the dynamic heat capacity given in eq. 8 is generally adopted in the literature (cf., e.g., refs. ${ }^{12,56}$ ). The desired oscillations of the kinetic temperature can be generated by perturbing the thermostat temperature $T_{\mathrm{i}}$ as ${ }^{61}$

$$
T_{\mathrm{i}}(t)=T_{\mathrm{i} 0}+\varepsilon e^{\mathrm{i} \omega t}
$$

with $\varepsilon \rightarrow 0$. The $T_{\mathrm{i}}$-oscillations lead to $\langle T(t)\rangle=T_{\mathrm{i} 0}+T_{\omega} e^{\mathrm{i} \omega t}$ and $\langle E(t)\rangle=\bar{E}+E_{\omega} e^{\mathrm{i} \omega t}$, so eventually $c_{v}^{*}(\omega)$ can be obtained using eq. 8 . By contrast, the heat capacity $c_{v 0}^{*}(\omega)$ defined by the r.h.s. of eq. 2 with $c_{v 0}(t)$ instead of $c_{v}(t)$ is equal to $c_{v 0}^{*}(\omega)=\frac{1}{N} \frac{E_{\omega}}{\varepsilon}$. Generally $\langle T(t)\rangle$ is not equal to $T_{\mathrm{i}}(t)$ and $T_{\omega} \neq \varepsilon$, so $c_{v 0}^{*}(\omega) \neq c_{v}^{*}(\omega)$. The inadequacy of eq. 5 (reflected in a deviation of $c_{v 0}(t)$ 
from $c_{v}(t)$ stemming from the difference between $c_{v 0}^{*}(\omega)$ and $\left.c_{v}^{*}(\omega)\right)$ is therefore due to thermal inertia of the system.

According to the FDT ${ }^{1-4,28}$ such a perturbation, eq. 9, leads to the following response of a variable $X, \Delta X=X(t)-X(-\infty)$ :

$$
\langle\Delta X(t)\rangle=\frac{N}{T^{2}} \int_{-\infty}^{t}\left[C_{X E}(0)-C_{X E}\left(t-t^{\prime}\right)\right] \mathrm{d} T_{i}\left(t^{\prime}\right)
$$

leading to

$$
\left\langle X_{\omega}\right\rangle=\frac{\mathrm{i} \omega \varepsilon N}{T^{2}} \int_{0}^{\infty}\left[C_{X E}(0)-C_{X E}(t)\right] e^{-\mathrm{i} \omega t} \mathrm{~d} t
$$

where $T=T_{i 0}$ and

$$
C_{X Y}(t)=\left\langle\delta X\left(t+t^{\prime}\right) \delta Y\left(t^{\prime}\right)\right\rangle / N
$$

is the time-correlation function. Using eq. 8 and eq. 11 for $Y=E, X=E$ and $X=T$ yields:

$$
c_{v}^{*}(\omega)=\left[c_{v s}-C_{E}(\omega) / T^{2}\right] /\left[1-N C_{T E}(\omega) / T^{2}\right]
$$

Here $c_{v s}=C_{E}(t=0) / T^{2}, C_{E}(\omega) \equiv C_{E E}(\omega)$, and the frequency-dependent correlation functions are defined according to the following equation (cf. eq. 2)

$$
C_{X Y}(\omega)=\mathrm{i} \omega \int_{0}^{\infty} C_{X Y}(t) e^{-\mathrm{i} \omega t} \mathrm{~d} t
$$

which resembles both Laplace and Fourier transforms (it will be called ' $\omega$-transform' in what follows). The time-dependent $c_{v}(t)$ can be obtained using eqs. 2,13 . Obviously $c_{v}^{*}(\omega)$ is different from

$$
c_{v 0}^{*}(\omega)=c_{v s}-C_{E}(\omega) / T^{2}
$$

(cf. eq. 5), so $c_{v}(t) \neq c_{v 0}(t)$.

The crucial point is that while the correlation functions $C_{E}(t)$ and $C_{T E}(t)$ do depend on the thermostat properties (like the parameter $Q$ ), the function $c_{v}^{*}(\omega)$ need not: it may reflect the original system properties. This statement can be considered as the following Ansatz:

Response functions defined in terms of physical variables of the original system must be universal, independent of the thermostat parameters in the thermodynamic limit.

The universality of response functions stated in the Ansatz is not entirely self-evident. Let us consider specifically the heat capacity $c_{v}(t)$. On the one hand, $c_{v}(t)$ defines the kinetic temperature response $\delta T(t)$ to small heats $\mathrm{d} q\left(t^{\prime}\right)$ injected into the kinetic degrees of freedom at $t^{\prime}<t$ (the total heat transmitted by the time $t$ is $\left.q(t)=\int_{-\infty}^{t} \mathrm{~d} q\left(t^{\prime}\right)\right)$. The injected heat can be a result $\left(\mathrm{d} q_{\text {ext }}\right)$ of an 
external perturbation of particle velocities (cf. eq. 16 below), or it can come from the thermostat $\left(\mathrm{d} q_{i}\right)$. It is a natural idea to assume that $\delta T(t)$ depends only on the total heat $q\left(t^{\prime}\right)=q_{\text {ext }}\left(t^{\prime}\right)+q_{i}\left(t^{\prime}\right)$, so that the two functions, $\delta T(t)$ and $q\left(t^{\prime}\right)$ are related in a universal linear fashion:

$$
q(t)=\int_{-\infty}^{t} N c_{v}\left(t-t^{\prime}\right) \frac{\mathrm{d} \delta T\left(t^{\prime}\right)}{\mathrm{d} t^{\prime}} \mathrm{d} t^{\prime}
$$

The Ansatz therefore says that it does not matter which source the heat is coming from (external or from the thermostat), what matters is its amount (and its time dependence). In other words, once a small heat perturbation $q(t)$ is imposed, the process of heat transfer from purely kinetic to vibrational and configurational degrees of freedom must depend solely on the physical system properties (particle interactions). On the other hand, a strict validity of this universality property requires certain conditions. Note first that we must consider here a 'decent' thermostat that just weakly perturbs the particle dynamics: the thermostat related velocity perturbation must be much smaller than the thermal velocity of a particle (cf. part (ii) of sect. VII B for details). It is also assumed that the external heat is transmitted by velocity perturbations (cf. eq. 16) with the same relative increment $(\varepsilon / d)$ for all the particles. It is also important to demand that the mechanism of heat transmission from the thermostat to the system must be similar (i.e., by perturbations of particle velocities providing uniform heating of the system ${ }^{62}$; a more detailed discussion of the sufficient conditions is delegated to section VIIB). Under these conditions the properly defined isochoric heat capacity (cf. eq. 8) is expected to be ensemble- and thermostat-independent.

The above Ansatz has non-trivial implications, as we show below. Importantly, we also provide an evidence (both analytical and from simulations) that this Ansatz is valid in the thermodynamic limit for a range of thermostatted systems as clarified in section VII B.

The above Ansatz can be used to get relations between correlation functions. To this end let us consider a direct perturbation when all particle velocities are slightly increased at $t=0$ :

$$
\underline{v} \rightarrow \underline{v}(1+\varepsilon / d)
$$

Applying FDT again we find the mean linear response of a variable $X$ :

$$
\langle\Delta X(t)\rangle=\frac{N^{2} \varepsilon}{T} C_{X T}(t), t \geq 0
$$

Using the above relation for $X=E$ and $X=T$, eq. 3 with $q(t)=\Delta E(t)$, and eq. 2 we get

$$
c_{v}^{*}(\omega)=N C_{E T}(\omega) / C_{T}(\omega)
$$


where $C_{T}(\omega) \equiv N^{2} C_{T T}(\omega)$. Again we see that $c_{v}^{*}(\omega)$ is expressed in terms of 2 correlation functions. Finally, using both eqs. 13 and 18 we obtain ${ }^{63}$ :

$$
C_{T}(\omega)\left[c_{v s}-C_{E}(\omega) / T^{2}\right]=N C_{T E}(\omega)\left[1-N C_{T E}(\omega) / T^{2}\right]
$$

Note that condition (i) of sect. VII B is required for validity of the above equation. In this case eqs. 13 and 18 define the same response function. Thus, eq. 19, which formally comes from the Ansatz, is expected to be rather generally applicable to canonical thermostatted systems (cf. section VII B for more precise criteria of its applicability; generalization of eq. 19 for some noncanonical thermostats is given in section VII A). Eq. 19 shows that only 2 correlation functions of $T$ and $E$ are independent. In the next section we rigorously prove this relation for the $\mathrm{NH}$ thermostat and show that, surprisingly, the universal $c_{v}(t)$ can be expressed in terms of a single non-universal correlation function (like $C_{T}(t)$ or $C_{E}(t)$ ) in this case.

\section{HEAT CAPACITY FOR THE NH THERMOSTAT}

One of the most popular thermostats is the canonical 'Nosé-Hoover' (NH) thermostat ${ }^{25-27,33,35}$ which is also a commonly used tool to investigate supercooled liquids (see e.g. refs. ${ }^{5-7,22}$ ). It is implemented in the well-known MD software package LAMMPS. ${ }^{32}$ The basic properties of the classical NH thermostat (corresponding to 'tchain $=1$ ' in LAMMPS) are outlined in Appendix B. Eq. B2 there implies that

$$
\dot{E}=-2 \xi K
$$

where $\xi$ is the friction parameter of the $\mathrm{NH}$ thermostat. Using eqs. 20, B3 one finds

$$
\dot{E}=-g \xi T_{i}-\frac{Q}{2} \frac{\partial}{\partial t}\left(\xi^{2}\right)
$$

leading to $\langle\xi\rangle=0$ (as long as the total energy is bounded) and the conservation law:

$$
E(t)+g T_{\mathrm{i}} \int \xi \mathrm{d} t+\frac{Q}{2} \xi^{2}=\text { const }
$$

The typical energy fluctuation is $\delta E \sim T \sqrt{N c_{v s}} \sim T \sqrt{N}$ (cf. eq. 1), while $\frac{Q}{2} \xi^{2} \sim T$ (cf. eq. B7). Therefore, the last term on the 1.h.s. of eq. 22 can be neglected for $N \gg 1$, and so

$$
E(t) \simeq \text { const }-g T_{\mathrm{i}} \int \xi \mathrm{d} t
$$


Recalling also eq. B3 we arrive at a linear relation between fluctuations of energy and temperature considered as functions of time (note that $\ddot{E}(t)$ is a linear functional of $E(t))^{64}$ :

$$
\ddot{E}(t)=-\frac{g^{2} T_{\mathrm{i}}}{Q}\left[T(t)-T_{\mathrm{i}}\right]
$$

Hence,

$$
N C_{T E}(t)=-k \frac{\partial^{2}}{\partial t^{2}} C_{E}(t), C_{T}(t)=-k N \frac{\partial^{2} C_{T E}}{\partial t^{2}}=k^{2} \frac{\partial^{4}}{\partial t^{4}} C_{E}(t)
$$

where $k=\frac{2}{d} \frac{1}{\omega_{Q}^{2}}, \omega_{Q}=\sqrt{2 g T_{\mathrm{i}} / Q}$ is the Nosé-Hoover frequency (cf. Appendix B). All energy correlation functions (of $E, K, U=E-K$ ) can be thus expressed in terms of just one function. Note also their relation with $\xi$-fluctuations:

$$
N C_{T E}(t)=T_{\mathrm{i}} Q C_{\xi \xi}(t)
$$

where $C_{\xi \xi}(t)=\left\langle\xi\left(t+t^{\prime}\right) \xi\left(t^{\prime}\right)\right\rangle, C_{\xi \xi}(0)=T_{\mathrm{i}} / Q$. In the frequency domain (cf. eq. 14) the above relations read:

$$
\begin{gathered}
N C_{T E}(\omega)=\frac{2}{d} \frac{\omega^{2}}{\omega_{Q}^{2}}\left[C_{E}(\omega)-T^{2} c_{v S}\right] \\
C_{T}(\omega)=\frac{2}{d} \frac{\omega^{2}}{\omega_{Q}^{2}}\left[N C_{T E}(\omega)-T^{2}\right]
\end{gathered}
$$

On using eqs. 25, 26 one can easily show that eq. 19 is identically satisfied thus supporting its generality. On the other hand, this fact also demonstrates that the two definitions of $c_{v}^{*}(\omega)$ given by eqs. 13 and 18 are equivalent thus backing the idea of universality of the heat-capacity functions $c_{v}(t)$ and $c_{v}^{*}(\omega)$.

Using eqs. 13, 25 we find

$$
1 / c_{v}^{*}(\omega)=1 / c_{v 0}^{*}(\omega)+\frac{2}{d} \frac{\omega^{2}}{\omega_{Q}^{2}}
$$

where $c_{v 0}^{*}(\omega)$ is defined in eq. 15. Eq. 27 allows to calculate the frequency-dependent heat capacity for the NH thermostat based solely on the energy autocorrelation function. It shows that $c_{v}^{*}(\omega) \simeq$ $c_{v 0}^{*}(\omega)$ for $\omega \ll \omega_{Q}$. In terms of the real-time functions it gives (on recalling eq. 5):

$$
c_{v}(t) \simeq c_{v 0}(t)=c_{v s}-\frac{1}{T^{2}} C_{E}(t), t \gg 1 / \omega_{Q}
$$

By virtue of eq. 27 a distinction between $c_{v}(t)$ and $c_{\nu 0}(t)$ disappears in the limit $\omega_{Q} \rightarrow \infty$ corresponding to a vanishing thermal mass $(Q \rightarrow 0)$. In the general case, as we know already (cf. Fig. 1), $c_{v 0}(t)$ depends on $Q$ at short $t$ in contrast to $c_{v}(t)$ which must be universal and $Q$-independent, being a physical property of the original energy-conserving system (cf. the Ansatz). 
Note that by definition

$$
c_{v}(t=0)=c_{v}^{*}(\omega \rightarrow \infty)=d / 2
$$

reflecting an obvious instant response of kinetic energy to a temperature increase. By contrast, $c_{v 0}(t=0)=0$. Having in mind the universality of $c_{v}$ it may be useful to express $c_{v 0}^{*}$ in terms of $c_{v}^{*}$ :

$$
c_{v 0}^{*}(\omega)=c_{v}^{*}(\omega) /\left[1-\frac{2}{d} \frac{\omega^{2}}{\omega_{Q}^{2}} c_{v}^{*}(\omega)\right]
$$

This equation shows, in particular, how $c_{v 0}^{*}$ depends on the Nosé-Hoover frequency $\omega_{Q}$. For example, for a system without potential energy (like a system of hard spheres) $c_{v}^{*}(\omega)=d / 2$. On using eq. 29 it leads to

$$
c_{\nu 0}(t)=\frac{d}{2}\left[1-\cos \left(\omega_{Q} t\right)\right]
$$

which shows infinitely persisting oscillations. Alternatively, eqs. 26, 25 allow to express $c_{v}^{*}(\omega)$ in terms of the temperature autocorrelation function. The result is

$$
c_{v}^{*}(\omega)=\frac{d}{2} \frac{\omega_{Q}^{2}}{\omega^{2}}+\frac{T^{2}}{C_{T}(\omega)}
$$

The above equation generalizes the classical relation valid for a canonical ensemble with energyconserving dynamics ${ }^{12,34,36}$

$$
c_{v}^{*}(\omega)=T^{2} / C_{T}(\omega)
$$

to the case of the Nosé-Hoover thermostat. ${ }^{65}$ The two equations (eq. 30 and 31) obviously coincide in the limit of very large thermal mass, $Q \rightarrow \infty$. Indeed, the Nosé-Hoover thermostat with $Q \rightarrow \infty$ conserves the total energy $E$ for finite time-intervals.

The asymptotic behaviors of the correlation functions at low and high frequencies (obtained using the general equations derived in this section) and other useful correlation properties are summarized in Appendix D.

\section{HOW TO AVOID THE TODA DEMON: THE QUESTION REVISITED}

The total energy or temperature of a classical system with NH thermostat often show persisting oscillations. This problem is well-known ${ }^{2,22,35}$; it is also illustrated in Fig. 1 . The frequency $\omega^{*}$ and the relaxation rate $\gamma^{*}$ of these oscillations strongly depend on the thermostat mass $Q$. In some cases the relaxation time $1 / \gamma^{*}$ gets extremely long, which deteriorates the velocity equilibration. 
This wild oscillation effect was analyzed in ref. ${ }^{35}$ (where it was labeled as being due to a 'demon' of a Toda oscillator). Below we revisit this effect based on eqs. 29, 30 which allow to get the energy and temperature correlation functions, $C_{E}(t)$ and $C_{T}(t)$, once the time-dependent heat capacity $c_{v}(t)$ is known. We demonstrate analytically how the physical response function $c_{v}(t)$ showing no oscillations can lead, by virtue of the $\mathrm{NH}$ thermostatting, to oscillating $C_{E}(t)$ and $C_{T}(t) .66$

Let us consider, for example, eq. 30. In effect, it defines the $\omega$-transform of $C_{T}(t)$ in terms of the $\omega$-transform of $c_{v}(t)$ :

$$
C_{T}(\omega)=T^{2} /\left(c_{v}^{*}(\omega)-\frac{d}{2} \frac{\omega_{Q}^{2}}{\omega^{2}}\right)
$$

Doing the inverse $\omega$-transform one can get $C_{T}(t)$ as a sum of exponentials with complex decay rates $s_{i}=\mathrm{i} z_{i}$, where $z_{i}$ are the complex frequencies corresponding to the poles in the r.h.s. of eq. 32 . In other words, $z_{i}$ are the roots of the characteristic equation

$$
\frac{2}{d} z^{2} c_{v}^{*}(z)-\omega_{Q}^{2}=0
$$

where $z=\omega+\mathrm{i} \gamma$ (the same characteristic equation also comes from eq. 29). Generally all the roots of eq. 33 are complex with positive $\mathfrak{I}(z) \equiv \gamma>0 .{ }^{67}$ The slowest relaxation mode corresponds to a pair of roots, $z^{*}= \pm \omega^{*}+\mathrm{i} \gamma^{*}$, with the lowest $\gamma=\gamma^{*}$. Typically $\gamma^{*}$ is small compared to $\omega^{*}$, so a perturbation approach can be used. For $\omega_{Q} \ll 1 / \tau_{\max }$, where $\tau_{\max }$ is the longest relaxation time (associated with $c_{v}(t)$ or $C_{T}(t)$ ), eq. 33 gives:

$$
\omega^{*} \simeq \omega_{Q} / \sqrt{2 c_{v S} / d}, \quad \gamma^{*} \simeq \eta_{E} \omega_{Q}^{2} / 2
$$

where $\eta_{E}$ is a universal $Q$-independent constant defined in eqs. D4, D9.

In the opposite regime, $\omega_{Q} \gg 1 / \tau$, where $\tau$ is the shortest relaxation time (of $c_{v}(t)$ ), the characteristic equation gives:

$$
\omega^{*} \simeq \omega_{Q}, \quad \gamma^{*} \simeq \eta\left(\omega^{*}\right) \omega^{* 2} / 2
$$

where the function $\eta(\omega)$ generalizes the constant $\eta_{E}=\eta(0)$ :

$$
\eta(\omega)=\int_{0}^{\infty}\left[c_{v s}-c_{v}(t)\right] \cos (\omega t) \mathrm{d} t
$$

(The two asymptotics given in eqs. 34, 35 for the oscillation frequency $\omega^{*}$ were obtained by Nosé ${ }^{26}$.) With smooth analytical $c_{v}(t)$, the factor $\eta\left(\omega^{*}\right)$ becomes exponentially small for $\omega^{*} \gg$ 
$1 / \tau$. This leads to extremely long-time damping of temperature oscillations in the high- $\omega_{Q}$ regime. $^{35}$

Thus, $\gamma^{*}$ decreases at both high and low $\omega_{Q}$, so the optimal $\omega_{Q}=\omega_{Q}^{*}$ (corresponding to the highest $\left.\gamma_{\max }=\gamma^{*}\left(\omega_{Q}^{*}\right)\right)$ is expected in the regime $\omega_{Q} \sim 1 / \tau$. To further justify this conclusion we consider below a model example assuming that the time-dependent heat capacity can be approximated with two exponentials:

$$
c_{v}(t)=\frac{d}{2}\left[1+A\left(1-e^{-\alpha t}\right)-A^{\prime}\left(1-e^{-\alpha^{\prime} t}\right)\right]
$$

with $A^{\prime}=A / \kappa, \alpha^{\prime}=A \kappa$ (so that $\mathrm{d} c_{v}(t) / \mathrm{d} t=0$ at $t=0$ ), and $c_{v s}=(d / 2)\left(1+A-A^{\prime}\right)$. We set the static heat capacity to $c_{v s}=d$ which is close its values for the $2 \mathrm{D} \mathrm{pLJ}$ system described in Appendix A. The relevant $\omega^{*}+\mathrm{i} \gamma^{*}$ was obtained by solving eq. 33 .

The dependencies of $\gamma^{*} / \alpha$ vs. $\omega_{Q} / \alpha$ for $\kappa=2$ and $\kappa=5$ are shown in Fig. 2a (the curves are independent of the basic relaxation rate $\alpha$ which is set to $\alpha=1$ for simplicity). A rather pronounced peak, $\gamma_{\max }=\gamma^{*}\left(\omega_{Q}^{*}\right)$, is visible in both cases at $\omega_{Q}=\omega_{Q}^{*}$. Clearly $\gamma^{*}$ gets very small for $\omega_{Q} \gg \omega_{Q}^{*}$ and $\omega_{Q} \ll \omega_{Q}^{*}$ : the Toda demon works there. Based on Fig. 2 and other results for this model we found that the decay rate $\gamma_{\max }$ and the optimal $\omega_{Q}^{*}$ can be approximated as:

$$
\gamma_{\max } \sim\left(2 c_{v s} / d-1\right) /\left(2 t_{1 / 2}\right), \omega_{Q}^{*} \sim 2 / t_{1 / 2}
$$

where $t_{1 / 2} \sim \tau$ is the median time for the $c_{v}(t)$ relaxation as shown in Fig. 2b. The Nosé frequency $\omega_{Q}=\omega_{Q}^{*}$ corresponds to the optimal parameter $Q=Q^{*} \sim(d / 2) N T t_{1 / 2}^{2}$. The recommended $\omega_{Q}^{*}$ given in eq. 38 is in harmony with conclusions of ref..$^{35}$ arguing that the optimal 'coupling' of the thermostat to the physical system is achieved at the inverse Nosé relaxation time $1 / \tau_{N}=\omega_{Q} / \sqrt{2} \sim$ $\omega_{E}$, where $\omega_{E}$ is the Einstein frequency.

For the $2 \mathrm{D} \mathrm{pLJ}$ system described in Appendix A we find: $t_{1 / 2} \approx 0.06$ for $T=0.4$, so $\omega_{Q}^{*} \sim 30$ (in $\mathrm{LJ}$ units). In practice, the median time can be estimated using the correlation function of potential energy, $C_{U}(t)$ (cf. eq. D10), which closely follows the dynamics of $c_{v}$ (see the next sections): $t_{1 / 2}$ can be defined by the condition $C_{U}\left(t_{1 / 2}\right)=C_{U}(0) / 2$. The function $C_{U}(t)$ may be obtained from a trial simulation run for a high $\omega_{Q} \gg 1 / \tau$, which means $\omega_{Q} \gtrsim 100$ for the system we simulated. ${ }^{68}$

\section{THE TIME-DEPENDENT $c_{v}$}

Eqs. 13, 27 or 30 allow to obtain the complex frequency-dependent function $c_{v}^{*}(\omega)$ based on the Laplace-Fourier-like $\omega$-transforms of correlation functions (cf. eq. 14). To this end one should 
first produce a time-dependent correlation function (say, $C_{E}(t)$ ) for a long time-interval, and then to calculate its $\omega$-transform according to eq. 14. However, calculation of $C_{E}(\omega)$ or $C_{T}(\omega)$ is generally a non-trivial task ${ }^{12}$ for several reasons: First, the power spectrum of, say, $E(t)$ is not enough

to get $C_{E}(\omega):\left\langle\left|E_{\omega}\right|^{2}\right\rangle$ defines only the imaginary part of this function. Therefore, it appears that evaluation of time-dependent correlation functions $\left(C_{E}(t), C_{T}(t)\right)$ with high accuracy and over many decades in time is a necessary intermediate step demanding a massive numerical work (in particular, in the case of supercooled liquids). Moreover, these functions typically show strong oscillations (see Fig. 3a,b) which may ruin the numerical precision of the $\omega$-transforms defined in eq. 14. Below we describe an efficient approach to calculate $c_{v}(t)$ avoiding such problems.

\section{A. The classical NH thermostat}

The starting point is that the correlation function of the potential energy $U, C_{U}(t) \equiv C_{U U}(t)$, exhibits much weaker oscillations than the kinetic energy (cf. Fig. 3a). Therefore it would be beneficial to use a relation between $c_{v}^{*}(\omega)$ and $C_{U}(\omega)$ (the $\omega$-transform of $C_{U}(t)$ defined in analogy with eq. 14). Such a relation can be found using eqs. 25, 26, 27; it reads:

$$
c_{v}^{*}(\omega)=(d / 2)(1+\varphi(\omega)+\psi(\omega))
$$

where

$$
\varphi(\omega)=\frac{2}{d}\left(c_{v s}-C_{U}(\omega) / T^{2}\right)-1, \quad \psi(\omega)=\frac{\omega^{2} \varphi(\omega)^{2}}{\omega^{2}(1-\varphi(\omega))-\omega_{Q}^{2}}
$$

Interestingly, it follows from these equations that $\varphi\left(\omega_{Q}\right)=0,{ }^{69}$ so

$$
C_{U}\left(\omega_{Q}\right)=T^{2}\left(c_{v s}-d / 2\right)
$$

This is a remarkable result. From the definition, eq. 14, we know that $C_{X Y}(t \rightarrow 0)=C_{X Y}(\omega \rightarrow \infty)$, so using eqs. D2, D3 we find

$$
C_{U}(\omega \rightarrow \infty)=T^{2}\left(c_{v s}-d / 2\right)
$$

and, hence, $C_{U}(\omega \rightarrow \infty)=C_{U}\left(\omega_{Q}\right)$. This relation provides yet another indication that fluctuations of potential energy are generally affected by the thermostat: otherwise we would have to conclude that $C_{U}(\omega)=$ const (since $\omega_{Q}$ can be varied independently of the original physical system properties). Eq. 41 also means that the power spectrum of $U=U(t)$ vanishes at $\omega_{Q}$ :

$$
P_{U}(\omega) \equiv \frac{1}{\pi} \int_{-\infty}^{\infty} C_{U}(t) e^{\mathrm{i} \omega t} \mathrm{~d} t=\frac{2}{\pi \omega} \mathfrak{I}\left[C_{U}(\omega)\right]=0 \text { at } \omega=\omega_{Q}
$$


Taking into account that $P_{U}(\omega) \geq 0$ (cf. Appendix C) we conclude that $P_{U}(\omega)$ has an absolute minimum at $\omega=\omega_{Q}$ meaning that fluctuations of $U$ are always suppressed near the Nosé frequency. Eqs. 39, 40 also show that $\psi(\omega) \ll \varphi(\omega)$ at low $\omega \ll \omega_{Q}$, hence

$$
c_{v}^{*}(\omega) \simeq c_{v s}-C_{U}(\omega) / T^{2}, \omega \ll \omega_{Q}
$$

Apart from $c_{v}^{*}(\omega)$, it is also of great interest to obtain the heat capacity function $c_{v}(t)$ in the time domain. To this end one may apply the inverse $\omega$-transform to $c_{v}^{*}(\omega)$. However, the whole procedure (to obtain $c_{v}(t)$ based on $C_{U}(t)$ ) then becomes rather complicated and can suffer from another problem near or below the glass transition temperature: In this case a significant relaxation of $C_{U}(t)$ occurs beyond the total simulation (sampling) time $\Delta t$ rendering it difficult to obtain $C_{U}(\omega)$ at low $\omega$. To deal with this matter one can try to extrapolate $C_{U}(t)$ at longer times, or to apply a low-frequency cut-off for $C_{U}(\omega)$. In both cases one has to rely on additional approximations to obtain $c_{v}(t)$.

Below we describe a different approach allowing to avoid these problems. The idea is to use a direct relation between $c_{v}(t)$ and $C_{U}(t)$ which can be derived from eqs. 39, 40. It reads:

$$
c_{v}(t)=\frac{d}{2}(1+\varphi(t)+\psi(t)), \varphi(t)=\frac{2}{d}\left(c_{v s}-C_{U}(t) / T^{2}\right)-1
$$

where $\psi(t)$ is a solution of the following equation

$$
\int_{0}^{t} \ddot{\varphi}\left(t-t^{\prime}\right)\left[\dot{\varphi}\left(t^{\prime}\right)+\dot{\psi}\left(t^{\prime}\right)\right] \mathrm{d} t^{\prime}=\omega_{Q}^{2} \psi(t)+\ddot{\psi}(t)
$$

and $\dot{\psi}(t) \equiv \mathrm{d} \psi(t) / \mathrm{d} t$, etc. This integral equation can be solved iteratively by small time steps starting from $t=0$ with the initial conditions $\psi(0)=0$ (coming from $\varphi(0)=0$ and $c_{v}(0)=$ $d / 2$ ) and $\dot{\psi}(0)=0$ (since both $\psi(t)$ and $\varphi(t)$ are even functions, cf. Appendix C). Remarkably, eqs. 43, 44 show that $c_{v}(t)$ is totally independent of the behavior of $C_{U}\left(t^{\prime}\right)$ at longer times, $t^{\prime}>t$. All 3 functions $c_{v}(t), \varphi(t)$ and $\psi(t)$ are also invariant with respect to a constant shift of $C_{U}(t)$, $C_{U}(t) \rightarrow C_{U}(t)+$ const, which may result from relaxation processes beyond the total sampling time.

For long $t$ the $\psi(t)$-term in $c_{v}(t)$ becomes negligible, so

$$
c_{v}(t) \simeq c_{v s}-C_{U}(t) / T^{2}, t \gg 1 / \omega_{Q}
$$

in agreement with eq. 42 . We used eq. 44 to calculate $c_{v}(t)$ of the model system (cf. Appendix A) for different thermal mass $Q$ at the same $T=0.4$. The results shown in Fig. 4 confirm our idea that $c_{v}(t)$ must not depend on $Q$. We also found that eq. 45 is valid (with relative error $\lesssim 5 \cdot 10^{-4}$ ) for $t \omega_{Q} \gtrsim 100$. 


\section{B. Canonical thermostats}

In the previous sections we considered the general relations between the dynamic heat capacity and the energy correlation functions, eqs. 27, 30, 39, 40, 43, 44, valid for classical NH thermostatted systems (with a single $\mathrm{NH}$ thermostat). We are now in a position to extend the analysis to a wider range of canonical systems. Such systems include, in particular, the 'NH chain' thermostatting mechanism ${ }^{38,39}$ implemented in LAMMPS ${ }^{32}$ and the stochastic Langevin thermostat ${ }^{22}$ (cf. sect. VII B). As discussed in sections V, VIA the energy and temperature correlation functions typically show unwanted persistent oscillations which render less precise numerical transformations of the correlation functions and slow down equilibration of the systems with single $\mathrm{NH}$ thermostat. The 'NH chain' is a general approach to suppress these adverse oscillation effects as illustrated in Fig. 3 (compare its parts (a) and (c)). From Fig. 3(c) it is also clear that the general approach to deal with fluctuations of the potential energy $U$ (cf. the previous section) is also efficient with NH chains.

The goal therefore is to get a general expression (not involving any thermostat parameters) for $c_{v}^{*}(\omega)$ in terms of $U$-correlations. Clearly, it is impossible to directly relate $c_{v}(t)$ with $C_{U}(t)$ as the very existence of such a relation would imply that $C_{U}(t)$ is thermostat-independent which is not the case. However, the dynamic heat capacity can be defined in terms of 2 correlation functions, $C_{U}(\omega)$ and $C_{U K}(\omega)$. Using the general eqs. $13,18,19$ we obtain ${ }^{70}$

$$
c_{v}^{*}(\omega)=\frac{d}{2}+\frac{d}{2} \frac{c_{v s}-d / 2-C_{U}(\omega) / T^{2}}{d / 2-C_{U K}(\omega) / T^{2}}
$$

In practice, both correlation functions, $C_{U} / T^{2}$ and $C_{U K} / T^{2}$, get small for $t \gg \tau$ (this regime corresponds to $\omega \tau \ll 1$ ), so the above equation can be simplified as

$$
c_{v}^{*}(\omega) \simeq c_{v s}-C_{U}(\omega) / T^{2}+(2 / d) c_{v s p} C_{U K}(\omega) / T^{2} \text { for } \omega \tau \ll 1
$$

Here $c_{v s p}=c_{v s}-d / 2$ is the potential energy contribution to the static heat capacity.

While eq. 46 defines the dynamic (frequency-dependent) heat capacity, it can be also transformed to the time-domain in analogy with eqs. 43,44 (here $\left.\dot{c}_{v}(t) \equiv \mathrm{d} c_{v}(t) / \mathrm{d} t\right)$ :

$$
c_{v}(t)=c_{v s}-\frac{1}{T^{2}} C_{U}(t)+\frac{2}{T^{2} d} \int_{0}^{t} \dot{c}_{v}\left(t^{\prime}\right) C_{U K}\left(t-t^{\prime}\right) \mathrm{d} t^{\prime}
$$

This equation has a unique and stable solution with $c_{v}(0)=d / 2$ and $\dot{c}_{v}(0)=0$ (the latter condition comes from $C_{U K}(0)=0$ for canonical systems). It can be found iteratively like for eq. 44 . The 
stability of eq. 48 is addressed in Appendix C. For $t \gg \tau_{\max }$ the integral in the r.h.s. can be approximated as

$$
\left[c_{v s}-c_{v}(0)\right] C_{U K}(t)
$$

leading to

$$
c_{\nu}(t) \simeq c_{v s}-\frac{1}{T^{2}} C_{U}(t)+\frac{2}{d} c_{v s p} \frac{1}{T^{2}} C_{U K}(t), t \gg \tau_{\max }
$$

We solved eq. 48 feeding it with the simulated correlation functions obtained for the model of Appendix A using NH chain thermostat $\left(\right.$ tchain $=3$ ) for $T=0.4, Q=4 / 3$ (corresponding to $\omega_{Q} \approx$ 110 , which is about 4 times larger than the optimal $\omega_{Q}^{*} \sim 30$ considered in section $\mathrm{V}$ ). The results for both single $\mathrm{NH}$ and $\mathrm{NH}$ chain thermostats are shown in Fig. 5. The two curves practically coincide in the whole time-range, the relative difference being extremely small, $\lesssim 3 \cdot 10^{-4}$. This discrepancy is rather close to the theoretical limit of precision which is $\sim 1 / N$. The Nosé-Hoover chain algorithm is known to produce ergodic behavior ${ }^{22}$ and correct heat capacity results ${ }^{40}$. The close agreement between the $c_{v}(t)$ functions obtained for tchain $=3$ and tchain $=1$ indicates that the classical NH method is also likely to ergodically sample the canonical ensemble, at least for the system we consider. The same conclusion was also drawn from simulations of supercooled polymer melts ${ }^{41}$ and films ${ }^{42}$ (using a Kremer-Grest-like bead spring model) where systems with a single NH thermostat were found to give dynamic properties in agreement with microcanonical simulations.

We also established that the approximate eq. 49 is valid with relative accuracy $\sim 10^{-4}$ for $t>1$ (for tchain $=3$ ). Rather amazingly it also turns out that another approximate eq. 45 is even more precise (for tchain $=3$ ): its relative error for $t>1$ is $\sim 5 \cdot 10^{-5} \cdot{ }^{71}$ Moreover, the latter equation works (for $Q=4 / 3)$ in the whole time-range $\left(0<t<10^{5}\right)$ with a reasonable relative error $\lesssim 1 \%$ (for both tchain $=1$ and tchain $=3$ ). Thus, it appears that for low $Q$ 's $\left(\omega_{Q} \tau \gg 1\right)$ the simple eq. 45 does a fairly good job.

Let us return to the general relation between the correlation functions, eq. 19. It can be easily transformed as

$$
C_{U K}(\omega)\left[\frac{d}{2} T^{2}-C_{U K}(\omega)\right]=C_{K}(\omega)\left[c_{v s p} T^{2}-C_{U}(\omega)\right]
$$

where $C_{K}(\omega)=C_{K K}(\omega)$ is the $\omega$-transformed autocorrelation function of the kinetic energy, $C_{K}(t)=(1 / N)\left\langle\delta K\left(t+t^{\prime}\right) \delta K\left(t^{\prime}\right)\right\rangle$. Eq. 50 shows that cross-correlations of kinetic and potential energies are generally defined by the corresponding autocorrelation functions. This equation must be valid for all $\mathrm{NH}$ or $\mathrm{NH}$-chain systems (with a relative error $\sim 1 / N$ ), and for other canonical 
thermostats (satisfying the conditions discussed in sect. VII B).

Noteworthily, Eq. 50 must be valid for any (complex) $\omega$ with $\mathfrak{I}(\omega) \leq 0$ including $\omega=-$ is with real $s$. We numerically verified this equation using the data obtained for the $2 \mathrm{D}$ pLJ system (described in Appendix A) with the NH-chain thermostat (LAMMPS parameter tchain = 3). We found that it works with accuracy $\sim 2 \%$ for $s$ in the range $20 \gtrsim s \gtrsim 2$. The error increases both at higher and lower $s$; it is due to somewhat oscillating character of the correlation functions and the smallness of $C_{K}(-\mathrm{i} s)$ at low $s$, while discretization errors (related to finite sampling time-step $\delta t$ ) are relevant at high $s$. Note that the accuracy of $c_{v}(t)$ obtained based on $C_{U}(t)$ and $C_{U K}(t)$ over the whole range of more than 5 decades in time is much higher (by a factor of $\sim 100$ ). This is because (i) we used the direct relation, eq. 48 , between $c_{v}(t)$ and time-dependent correlation functions, avoiding the $\omega$-transforms (cf. eq. 14), and (ii) the oscillating autocorrelation function of kinetic energy is not involved in eq. 48. Hence, the numerical scheme based on eq. 48 (involving $C_{U}$, $C_{U K}$ ) is preferable for 3 reasons: its high stability, its high precision due weak oscillations of input functions, and independence of the resultant $c_{v}(t)$ of the total sampling time.

\section{DISCUSSION}

\section{A. Non-canonical thermostats}

So far we implicitly assumed that the equilibrium distribution of the extended system in the phase space is canonical. ${ }^{22}$ Indeed, introducing eqs. 5 and 11 we referred to the generic FDT (a relevant argument can be found in section IV of ref. ${ }^{28}$ ) which assumes such canonical equilibrium distribution. Thus, eqs. 13, 18, 19 are applicable only to canonical thermostats. ${ }^{72}$

The canonical equilibrium distribution density in the phase space, $\rho_{0}(x, p)$, is an exponential function of the total energy $E$ only. The 'canonical thermostat' requirement may be weakened by allowing $\rho_{0}$ to depend on both the kinetic energy $K$ and the potential energy $U: \rho_{0}=f(K, U), E=$ $K+U$ with virtually arbitrary function $f$ (which of course must decrease exponentially, or faster, for high energies). In this more general case (which we refer to as a quasi-canonical ensemble) the static fluctuations of $K$ and $U$ may not obey the well-known relations with thermodynamic derivatives. Accordingly, the basic relations, eqs. 13, 18, 19, must be generalized to compensate for this effect. The main idea is simple: In the canonical case the initial (in the case of eq. 16) or 
terminal (in the case of eq. 4) perturbations can be described as

$$
\rho=\rho_{0}+\delta \rho=\rho_{0}\left(1+\varepsilon\left(X-X_{0}\right)\right)
$$

where $X_{0}$ is the averaged $X$ for the $\rho_{0}$-distribution, $\varepsilon$ is the perturbation magnitude and $X=\frac{N}{K_{i}} K$ or $\frac{1}{T_{i}^{2}} E$, respectively (here $\left.K_{i}=(d / 2) N T_{i}\right)$. In the non-canonical case the conjugated variable $X$ must be modified to provide the correct responses, $\Delta T$ and $\Delta E$ (or, equivalently, $\Delta K$ and $\Delta U$ ), to the perturbation. For example, the velocity perturbation of eq. 16 leads to: $\Delta T=\frac{2}{d} \varepsilon T, \Delta E=\varepsilon K=$ $\varepsilon N T, \Delta E / \Delta T=N d / 2$ at $t=0$. The $T_{i}$-perturbation (eq. 4) corresponds to $\Delta E / \Delta T=c_{v s} N$ at $t=\infty$. Thus

$$
\begin{gathered}
X=\frac{N}{K_{i}}(K-\beta E), \quad v \text {-perturbation } \\
X=\frac{1}{T_{i}^{2}}\left(E+\beta^{\prime} K\right), T_{i} \text {-perturbation }
\end{gathered}
$$

with

$$
\beta=\frac{C_{U K}}{C_{U E}}, \quad \beta^{\prime}=\frac{C_{E E}-\tilde{c}_{v S} C_{K E}}{\tilde{c}_{v S} C_{K K}-C_{E K}}
$$

where $\tilde{c}_{v s}=(2 / d) c_{v s}$ is the reduced static heat capacity and $C_{X Y} \equiv\langle\delta X \delta Y\rangle / N=C_{X Y}(t=0)$ are the static correlators.

After some algebra we get the following generalizations of eqs. 13, 18, 19:

$$
\begin{gathered}
c_{v}^{*}(\omega)=\frac{d}{2} \frac{C_{E K}(\omega)-\beta C_{E}(\omega)}{C_{K}(\omega)-\beta C_{K E}(\omega)} \\
c_{v}^{*}(\omega)=\frac{d}{2} \frac{\tilde{c}_{v S}-\alpha C_{E}(\omega)}{1-\alpha C_{K E}(\omega)} \\
\alpha\left[C_{E}(\omega) C_{K}(\omega)-C_{K E}(\omega) C_{E K}(\omega)\right]-\beta\left[C_{E}(\omega)-\tilde{c}_{v S} C_{K E}(\omega)\right]+C_{E K}(\omega)-\tilde{c}_{v S} C_{K}(\omega)=0
\end{gathered}
$$

where

$$
\alpha=\left(\tilde{c}_{v S}-1\right) / C_{U E}, \quad \beta=C_{U K} / C_{U E}
$$

Note that the above equations do not require time-reversibility of the dynamics. Apart from canonical systems, these equations are applicable, for example, to the Gaussian isokinetic (GIK) thermostat ${ }^{43-46}$ whose equilibrium distribution density $\rho_{0}$ is a function of $U$ and $K^{47}$. (Note, however, that eq. 51 is useless for the GIK systems: its r.h.s. gives $0 / 0$ since $K=$ const in this case.) Using eq. 52 we get a simple equation for the time-dependent heat capacity with a GIK thermostat:

$$
c_{v}(t)=c_{v s}-(d / 2) \alpha C_{E}(t)=c_{v s}-T^{-2} C_{U}(t)
$$


where $C_{U}(t)$ is defined in eq. D10 and we take into account that the equilibrium distribution of potential energy for a GIK system (but not the distribution density as a whole) is canonical ${ }^{47}$, so $C_{U}(t=0)=\left(c_{v s}-d / 2\right) T^{2}$.

\section{B. The region of applicability of the Ansatz and the general relations between response and correlation functions}

In section III we introduced the basic Ansatz stating the universality of response functions, i.e. their independence of the thermostatting method and the related coupling parameters. Justification of this principle for the $\mathrm{NH}$ dynamics is hinged on the idea that it does not matter how a particular $T$-perturbation is generated, by an external perturbation of particle velocities (cf. eq. (16)) or by the thermostat-related force, $-\xi p$ (cf. eq. (B2)): the ensemble-averaged energy-response must be the same (or, equivalently, the temperature response is defined by the amount of injected heat, no matter which source it comes from). This equivalence stems from the weakness of instantaneous temperature fluctuations $(\delta T \ll T)$ at equilibrium. The physical idea behind the Ansatz is thus similar to that underlying the famous random phase approximation (RPA) widely used in statistical physics.

Based on this Ansatz we obtained a number of the general relations, eqs. 13, 18, 19, 52, 51, 53, valid for canonical and quasi-canonical systems (the latter class is described in the previous section). Are there any general conditions for these equations to be asymptotically exact (in the thermodynamic limit)? The answer is yes: we believe that the following conditions are sufficient (although may be not necessary):

(i) The temperature control must be maintained by virtue of artificial friction forces depending on particle velocities. More precisely, the thermostat-related force $\underline{f}^{\prime}$ on a particle must depend on its momentum $\underline{p}$ only:

$$
\underline{f}^{\prime}=-\xi \underline{p}+\eta
$$

where $\xi$ is a constant or time-dependent damping coefficient, which must be the same for all particles, and $\eta$ is a stochastic force uncorrelated with particle positions and velocities.

The above condition is obviously valid for a range of thermostats including the deterministic $\mathrm{NH}^{25,26,33}$ and NH-chain ${ }^{38,39}$ ) thermostats with $\xi=\xi(t)$ and $\eta=0$, the stochastic Langevin dynamics $^{22}$ (with a single damping constant), and the Gaussian isokinetic (GIK) model ${ }^{43}$. Indeed, in section IV we proved that the general 'canonical' eq. 19 is valid for systems with the classical 
$\mathrm{NH}$ thermostatting mechanism. Its validity for the Langevin thermostat (with a single damping constant) can be proved in a similar way.

The requirement of equal damping parameter $\xi$ for all particles is essential as argued below. Consider a binary mixture (say, of large and small particles). Let us modify the NH dynamics by applying different damping to the two sorts of particles: say, $\xi_{\text {large }}=\xi$ and $\xi_{\text {small }}=\xi / 1000$. Obviously, it must lead to a much less efficient temperature regulation for small particles. Suppose in addition that interactional coupling between small and large particles is very weak. Then a steplike increase of the imposed temperature $T_{i}$ would result in a fast increase of the instantaneous $T$ for large particles, but (for a long time) in a little $T$-effect for the subsystem of small particles. These effects must lead to an incorrect overall response $c_{v}(t)$ at intermediate times (reflecting the properties of the large-particle subsystem only).

Note that while the above condition (i) may be sufficient for the validity of the general relationships like eqs. $19,50,53$, it does not guarantee that the response functions of the system are (almost) not perturbed by the thermostat effect. Another condition must be added to ensure the latter property:

(ii) The system/thermostat coupling must be weak at the particle level, so that it virtually does not affect the particle velocity autocorrelation function (or the mean-square displacement).

By weak coupling here we mean that the thermostat-related variation $\delta v$ of a particle velocity during collision/vibration time $\tau$ must be small: $\delta v \ll v$. This condition is always valid with $\mathrm{NH}$ dynamics ${ }^{25,33}$ : in this case $\delta v / v \lesssim \xi / \omega_{Q} \sim 1 / \sqrt{N}$ (cf. Appendix B, eq. B7 and the text below it) ${ }^{73}$, hence $\delta v / v \ll 1$ for $N \gg 1$. Therefore, eqs. 13, 18, 19 are valid with NH dynamics for any thermal mass $Q$, and the time-dependent heat capacity $c_{v}(t)$ does not depend on $Q$.

The situation is different with the Langevin dynamics: in this case $c_{v}(t)$ may depend on the damping parameter $\xi$. Yet, eq. 19, is always valid with the Langevin thermostat (the proof is similar to that given in sect. IV), and, on the other hand, the full universality of $c_{v}(t)$ (its independence of $\xi$ ) is approximately restored for weak enough $\xi$, in the regime $\xi \tau \ll 1$.

Finally, we note that the condition (i) is not really satisfied in the case of the dissipative particle dynamics (DPD) algorithm, where the frictional forces depend on the relative velocities of interacting particles ${ }^{10}$. In this case the dynamic heat capacity can be still obtained using eq. 13 (or, alternatively, eq. 48), but the result may depend on the strength of system/thermostat frictional coupling. Nevertheless we anticipate that the formalism developed in this paper may be approximately applicable for the momentum-conserving DPD simulations with weak frictional coupling. 


\section{SUMMARY AND CONCLUSIONS}

In this paper we demonstrated that correlation functions of large thermostatted systems (like, for example, supercooled liquids) generally depend on the temperature-control mechanism (see section II). More precisely, the idea that correlation functions obtained with different thermostats (for example, with $\mathrm{NH}$ and GIK methods) are the same ${ }^{33}$ (and are independent of the system/thermostat coupling strength) may be valid in some regimes (e.g. in the liquid state) and only for those variables whose fluctuations are essentially decoupled from temperature fluctuations (like, for example, the shear stress or a particle velocity component ${ }^{25}$ ). However, it is generally not valid for other variables like energy and its potential part, or pressure and its virial (excess) part: auto- and cross-correlation functions of these variables are perturbed by the thermostat and hence must depend on the thermostat parameters like the thermal mass $Q$ (for Nosé-Hoover simulations) or the friction constant (for Langevin simulations). In other words, these correlation functions are spoiled by the temperature regulation mechanism. This property is immanent in thermostatted systems; with respect to kinetic energy fluctuations it was recognized in refs. ${ }^{26,33,34}$.

By contrast, the properly-defined response functions (like the dynamic heat capacity $c_{v}^{*}(\omega)$ defined via eq. 8) must be universal, independent of the thermostat properties (for 'decent' thermostats described in sect. III). We formulated this statement as a physical Ansatz (section III) and argued, focusing on $c_{v}^{*}(\omega)$, that it is valid for a range of thermostatting mechanisms including the Nosé-Hoover (NH), NH-chain, and Gaussian isokinetic (GIK) thermostats. The whole class of eligible thermostats is outlined in sections VII A, VII B.

The fact that the correlation functions of energy or temperature do depend on the thermostat properties together with universality of $c_{v}^{*}(\omega)$ means that there is no general relation between $c_{v}^{*}(\omega)$ and a single correlation function. In fact, the known FDT relations ${ }^{28,34}$, eqs. 5, 31, are not really applicable generally: eq. 31 is valid only for microcanonical (NVE) ensemble (which is not a thermostatted system), while eq. 5 is never valid at short times.

To fill the gap for the classical NH thermostatting mechanism, we obtained an exact expression for $c_{v}^{*}(\omega)$ in terms of the $\omega$-transform (cf. eq. 14) of the potential energy autocorrelation function $C_{U}(t)$ (cf. eqs. 39, 40). Moreover, we found a general relation, eq. 50, between 3 correlation functions: $C_{U}(t)$, the autocorrelation function of the kinetic energy, $C_{K}(t)$, and the cross-correlation function, $C_{U K}(t)$, deriving it directly from the Ansatz for canonical thermostats. Importantly, the validity of this relation, eq. 50, was also proved based on the $\mathrm{NH}$ dynamical equations and 
validated numerically for the NH-chain dynamics (cf. sections IV, VIB). An even more general equation for $c_{v}^{*}(\omega)$ in terms of two correlation functions, $C_{U}$ and $C_{U K}$ (eq. 46) is derived in sect. VIB. This equation is applicable to all canonical thermostats respecting the criteria discussed in section VII B.

The whole theory was also generalized (cf. section VII A) to non-canonical thermostats with arbitrary $U$ - and $K$-fluctuations and their cross-correlations. On this basis we derived a simple general equation defining $c_{v}(t)$ for the GIK thermostat (cf. eq. 55).

Furthermore, we derived useful direct relations between $c_{v}(t)$ and the time-dependent correlation functions, eqs. $43,44,48$. These equations possess the following feature: $c_{v}\left(t^{\prime}\right)$ obtained on their basis in the range $0<t^{\prime}<t$ does not depend on the behavior of the energy correlation functions at $t^{\prime}>t$, which allows to avoid long-time extrapolations of correlation functions. Solving eqs. 43, 44 using simulation data for the NH dynamics (on the 2D pLJ supercooled liquid described in Appendix A) with different values of thermal mass parameter $Q$ we demonstrated universality of the time-dependent heat capacity $c_{v}(t)$ : the resultant $c_{v}(t)$ proved to be independent of $Q$ (cf. Fig. 4). Moreover, solving eqs. 43, 44, 48 for the lowest $Q$ we obtained $c_{v}(t)$ over many decades in time based on correlation functions of the potential energy. The function $c_{v}(t)$ was calculated twice using the data from 2 independent $\mathrm{NH}$ and $\mathrm{NH}$-chain simulations (LAMMPS parameter tchain $=1$ and 3, respectively). The two curves (shown in Fig. 5) coincide with remarkable precision (relative error $\left.\lesssim 3 \cdot 10^{-4}\right){ }^{74}$ In this figure one can observe a fast process (collisions/vibrations) at $t \sim 0.05$, then a weak overshoot and a shoulder followed by a broad terminal relaxation at $1 \lesssim t \lesssim 30$. Note that the $\alpha$-relaxation time derived from the shear-stress relaxation modulus (not shown) is $\tau_{\alpha} \sim 2$, so it seems that an even slower process is reflected in the transient heat capacity.

It is worth noting that all relations considered in the present paper are valid in the thermodynamic limit, for a finite $N$ their relative accuracy is $\sim 1 / N$.

In conclusion, let us emphasize the main achievements reported in the present paper:

(i) We derived a number of general relations between the universal transient heat capacity $c_{v}(t)$ (and its frequency-dependent transform, $c_{v}^{*}(\omega)$ ) and the non-universal (thermostat-dependent) energy correlation functions, valid both in frequency- and time-domains (eqs. 39, 40, 46 and 43, 44, 48, respectively) for a range of thermostatting mechanisms.

(ii) Equally general relations between the energy correlation functions are established for canonical (eq. 50) and non-canonical (eq. 53) thermostats.

(iii) We propose 3 approaches (altogether efficient) to increase the precision of $c_{v}(t)$ by avoiding 
the adverse effect of oscillations in the energy correlation functions: (a) To use low thermal mass parameter $Q$ corresponding to a high Nosé frequency $\omega_{Q}$ exceeding the highest relaxation rate of the system: $\omega_{Q} \gtrsim \omega_{Q}^{*}$, corresponding to $Q \lesssim(d / 2) N T t_{1 / 2}^{2}$ (cf. section V). (b) To use direct relations between $c_{v}(t)$ and time-dependent correlation functions, thus bypassing the inverse $\omega$ transforms which may be detrimental to numerical precision. (c) To employ eqs. 43, 44, 48 defining $c_{v}(t)$ in terms of the potential energy correlation functions, $C_{U}(t)$ and $C_{U K}(t)$, showing much weaker oscillations than the autocorrelation function $C_{E}(t)$ of the total energy $E$.

The ideas and approaches proposed in the present paper can be used to obtain many other response functions for variables whose fluctuations are correlated with $T$-fluctuations, in particular, the pressure relaxation modulus (dynamic compression modulus), the transient isobaric heat capacity $c_{p}(t)$, the time-dependent pressure response to a $T$-perturbation, and also the wave-vector $\underline{q}$-dependent generalizations of these and other response functions.

\section{Data availability}

The data that support the findings of this study are available from the corresponding author upon reasonable request.

\section{ACKNOWLEDGMENTS}

A grant of computer time at the HPC computing cluster of the University of Strasbourg is gratefully acknowledged. We also thank O. Benzerara and H. Meyer (all from Strasbourg) for assistance in some aspects of MD simulations and helpful discussions. L.K. was supported by a doctoral contract from the University of Strasbourg in the framework of the IRTG "Soft Matter Science".

\section{Appendix A: The model and simulation approach}

We study a 2-dimensional (2D) polydisperse system of $N=10^{4}$ Lennard-Jones (LJ) particles with the same mass $m_{p}{ }^{7,8,48}$. The particle diameters, $\sigma_{l}$ with $l=1, \ldots, N$, are uniformly distributed between $(1-\Delta) \bar{\sigma}$ and $(1+\Delta) \bar{\sigma}$ with $\Delta=0.2$ and $\bar{\sigma}$ being the mean diameter of the particles. The mean-square particle size is $\overline{\sigma^{2}}=\left(1+\Delta^{2} / 3\right) \bar{\sigma}^{2}$, leading to the size polydispersity index of $\delta_{\mathrm{p}}=$

$\overline{\sigma^{2}} / \bar{\sigma}^{2}-1=\Delta^{2} / 3$. Each pair of particles (of diameters $\sigma_{l}$ and $\sigma_{k}$ ), a distance $r$ apart, interacts with energy $u_{\mathrm{LJ}}(s)$, where $s=r / \sigma_{l k}, \sigma_{l k}=\left(\sigma_{l}+\sigma_{k}\right) / 2$ and the LJ potential $u_{\mathrm{LJ}}(s)=4 \varepsilon\left(s^{-12}-s^{-6}\right)$ 
was truncated at $s_{\text {cut }}=2^{7 / 6} \approx 2.24$ and shifted to avoid discontinuity $\left(u(s)=0\right.$ for $\left.s>s_{\text {cut }}\right)$. All quantities in the main text are given in LJ units: $m_{p}, \bar{\sigma}$, the energy $\varepsilon$, and the Boltzmann constant $k_{\mathrm{B}}$ are set to unity. In particular, the Lennard-Jones time $\tau_{\mathrm{LJ}}=\sqrt{m_{p} \bar{\sigma}^{2} / \varepsilon}=1$.

We used a standard molecular dynamics (MD) with velocity-Verlet algorithm and periodic boundary conditions as implemented in the LAMMPS code ${ }^{5,32}$. The system was equilibrated at constant pressure $P_{0}=2$ and $T=1$ (using a Nosé-Hoover thermostat and barostat) to prepare $m=100$ well-relaxed independent configurations. Starting from the initial temperature $T=1$ these configurations were then continuously cooled down at $P_{0}=2$ with rate $\mathrm{d} T / \mathrm{d} t=-10^{-5}$. These slow cooling runs allowed to determine the glass transition temperature, $T_{\mathrm{g}} \approx 0.26$, using a dilatometric criterion ${ }^{7,8}$. Each configuration was then tempered for $\Delta t_{1}=2 \cdot 10^{5}$ in the NPT ensemble at $P=P_{0}$ to further equilibrate the system and then for another $\Delta t_{1}$ in the canonical NVT ensemble (using Nosé-Hoover thermostat). Finally, the production runs (served to obtain all correlation functions) were performed during the total sampling time $\Delta t=10^{5}$ in the NVT ensemble. The MD step was $t_{M D}=0.001$ with tchain $=1$ (except for the analysis of $\bar{K}$, cf. end of Appendix D) and $t_{M D}=0.002$ with tchain $=3$. The linear dimension of the simulation box was $L \sim 100$, the system volume $V=L^{2}$ and the 2 -dimensional particle concentration $c_{0}=N / V \sim 1$.

\section{Appendix B: The Nosé-Hoover (NH) thermostat}

Consider a system of $N$ classical particles in a $d$-dimensional space, with mass $m_{i}$ for the $i$ th particle, $i=1, \ldots, N$. The system microstate in its phase space is given by the coordinates $x=\left\{x_{i \alpha}, i=1, \ldots, N, \alpha=1, \ldots, d\right\}$ and momenta $p=\left\{p_{i \alpha}=m_{i} v_{i \alpha}\right\}$ of all particles, where $x_{i \alpha}$ is Cartesian $\alpha$-coordinate of particle $i$ and $v_{i \alpha}$ is $\alpha$-component of its velocity. The canonical Hamiltonian dynamical equations for the system are

$$
\dot{x}=v, \quad \dot{p}=-\frac{\partial U}{\partial x}
$$

where $U=U(x)$ is its potential energy.

The classical NH thermostat involve an auxiliary variable $\xi$ and 2 additional parameters, the imposed temperature $T_{\mathrm{i}}$ and 'thermal mass' $Q^{22,25-27}$. The NH equations of motion are

$$
\begin{gathered}
\dot{x}=v, \quad \dot{p}=-\frac{\partial U}{\partial x}-\xi p \\
\dot{\xi}=\frac{g}{Q}\left(T-T_{\mathrm{i}}\right)
\end{gathered}
$$


where $T=T(t)$ is instantaneous temperature,

$$
T=\frac{2}{g} K, \quad K=\sum_{i \alpha} p_{i \alpha}^{2} /\left(2 m_{i}\right)
$$

and $g$ is the number of kinetic degrees of freedom, $g \simeq d N$ (see note ${ }^{60}$ after eq. 7). The $\mathrm{NH}$ approach is therefore equivalent to dealing with an extended Newtonian system whose phasespace is enriched by auxiliary variables. The $\mathrm{NH}$ thermostat provides reliable results for both equilibrium and non-equilibrium properties (like thermal conductivity) $33,40,49$.

Consider an element of the extended phase space $(x, p, \xi)$ transforming in time according to the dynamical equations B2, B3. Its volume $\Omega$ then changes with rate ${ }^{39}$

$$
\mathrm{d}(\ln \Omega) / \mathrm{d} t=-g \xi
$$

The above equation generalizes the Liouville's theorem on phase-volume conservation in Hamiltonian systems ${ }^{1,22}$ to the $\mathrm{NH}$ thermostatted systems.

The NH dynamics is time-reversible (as follows from eqs. B2, B3) and canonical. The latter feature means that if the initial distribution, $\rho=\rho(x, p, \xi)$, of an ensemble of NH systems in the extended phase space is

$$
\rho=\rho_{0}(x, p, \xi) \propto \exp \left(-\left(H+Q \xi^{2} / 2\right) / T_{\mathrm{i}}\right)
$$

where $H(x, p)=K(p)+U(x)$ is the total energy (the Hamiltonian), $\rho=\rho_{0}$ will remain unchanged in time. ${ }^{25,26}$ This feature comes directly from eqs. B5 and 22. It implies (assuming the ergodicity) that any initial distribution $\rho$ must evolve to $\rho_{0}(x, p, \xi)$ after a sufficiently long relaxation, so the equilibrium distribution (after reduction to the classical phase-space) is always canonical. In particular, at equilibrium $\langle K\rangle=g T_{\mathrm{i}} / 2$ and

$$
\left\langle\xi^{2}\right\rangle=T_{\mathrm{i}} / Q
$$

There are some concerns about possible lack of ergodicity for the NH dynamics. ${ }^{22,39}$ These concerns however seem to be exaggerated: there is no reason to expect that $\mathrm{NH}$ dynamics is less ergodic than, say, energy-conserving Newtonian dynamics if sampling is allowed for long enough time. The real problem with ergodicity is related to the emergence of slow configurational relaxation modes (say, due to vitrification) which are independent of the thermostatting approach. Here the challenge is to accelerate the configurational equilibration, and certain techniques were devised to this end ${ }^{23,50-54}$. 
Interestingly, eqs. B2, B3 imply that in an ideal system with $U \equiv 0$ both $\xi$ and $T$ would oscillate indefinitely with the Nosé frequency $\omega_{Q}=\left(2 g T_{\mathrm{i}} / Q\right)^{1 / 225,26}$. In some NH systems with particle interactions the temperature may still take a very long time to relax. ${ }^{35,55}$ However, this problem can be healed by tuning the thermal mass parameter $Q$ (to get the optimal $\omega_{Q} \sim 1 / \tau$, where $1 / \tau$ is the decay rate of a significant fast relaxation process ${ }^{35}$; cf. section $\mathrm{V}$ ), or by using chains of $\mathrm{NH}$ thermostats ${ }^{38}$. Yet another possibility is to prepare the initial independent configurations using a different, more efficient equilibration method (for example, MC dynamics ${ }^{9,50,54}$ ).

\section{Appendix C: Stability of eq. 48}

Let us first recall some general properties of the correlation functions $C_{X Y}(t)$ with $X, Y$ being linear combinations of potential $(U)$ and kinetic $(K)$ energies. These functions are even in time, so they can be represented as

$$
C_{X Y}(t)=\int_{0}^{\infty} P_{X Y}(\omega) \cos (\omega t) \mathrm{d} \omega
$$

with real $P_{X Y}(\omega)$. Moreover, for $Y=X$ the power spectrum $P_{X X}(\omega) \geq 0$ due to the WienerKhinchin theorem. The response function $c_{v}(t)$ is physically defined only for $t \geq 0$. However, it can be also defined mathematically using inverse Laplace transform of, say, eq. 46. This way $c_{v}(t)$ may be analytically continued to the region of negative $t$. It is easy to show that $c_{v}(-t)=c_{v}(t)$ (for example, using eq. 48), and that in analogy with eq. C1

$$
c_{v s}-c_{v}(t)=\int_{0}^{\infty} P_{c v}(\omega) \cos (\omega t) \mathrm{d} \omega
$$

where

$$
P_{c v}(\omega)=\frac{2}{\pi} \mathfrak{I}\left(\frac{c_{v s}-c_{v}^{*}(\omega)}{\omega}\right) \geq 0
$$

Note that $P_{c v}(\omega) \geq 0$ is due to the condition of non-negative energy dissipation (entropy production) $)^{15,34}$ :

$$
\mathfrak{I}\left(c_{v}^{*}(\omega)\right) \leq 0 \text { for real } \omega \geq 0
$$

The properties mentioned above are related to the fact that all poles of the expression in the r.h.s. of eq. 46 are located at $\mathfrak{I}(\omega) \geq 0$. To formally prove this statement it is enough to show that there are no solutions of equation

$$
C_{U K}(\omega)-d T^{2} / 2=0
$$


with $\mathfrak{I}(\omega)<0$ since all singularities of the function $C_{U}(\omega)$ in the numerator of eq. 46 are necessarily located in the semi-plane $\mathfrak{I}(\omega) \geq 0$ (as follows from eq. 14). Finally, we note that by virtue of eq. 50 the latter property (concerning the roots of eq. C5) is true if both $C_{K}(\omega)$ and $c_{v s p} T^{2}-C_{U}(\omega)=C_{U}(\omega \rightarrow \infty)-C_{U}(\omega)$ are nonzero for $\mathfrak{I}(\omega)<0$. Below (at the end of this section) we show how to prove the first part of the statement concerning $C_{K}(\omega)$. The second part concerning $C_{U}(\omega)$ can be proved in a similar way.

Let us now return to eq. 48. Stability of its solution at long times means that there is no exponentially increasing function $f(t), f(t) \propto \exp \left(s_{0} t\right)$ with $\mathfrak{R}\left(s_{0}\right)>0$, satisfying the corresponding homogeneous equation

$$
f(t)-\frac{2}{T^{2} d} \int_{0}^{t} \dot{f}\left(t-t^{\prime}\right) C_{U K}\left(t^{\prime}\right) \mathrm{d} t^{\prime}=0
$$

For long $t$ eq. C6 leads to

$$
1-\frac{2}{T^{2} d} s_{0} \int_{0}^{\infty} C_{U K}(t) e^{-s_{0} t} \mathrm{~d} t=0
$$

which, in turn, is equivalent to

$$
1-\frac{2}{T^{2} d} C_{U K}\left(\omega=-\mathrm{i} s_{0}\right)=0
$$

Clearly, eq. C7 is equivalent to eq. C5 with $\mathfrak{I}(\omega)<0$. As stated above there are no such solutions of eq. C5, hence the solutions of eq. 48 must be stable. Moreover, it is easy to show that if eq. C6 is imposed at all times, then Laplace transform of its solution $f(t)$ must be identically zero, hence $f(t) \equiv 0$. This proves that eq. 48 has a unique solution.

Let us now show that $C_{K}(z) \neq 0$ if $\mathfrak{I}(z)<0$, where $z$ is used instead of $\omega ; z=\omega_{0}-\mathrm{i} \gamma$ with real $\omega_{0}$ and real $\gamma>0$. Using eq. $\mathrm{C} 1$ we get $C_{K}(z)=-z I$, where

$$
I=\int_{0}^{\infty} P_{K}(\omega) \frac{z}{\omega^{2}-z^{2}} \mathrm{~d} \omega
$$

leading to

$$
\mathfrak{I}(I)=-\gamma \int_{0}^{\infty} P_{K}(\omega) \frac{\omega^{2}+|z|^{2}}{\left|\omega^{2}-z^{2}\right|^{2}} \mathrm{~d} \omega
$$

Recalling that $P_{K}(\omega) \geq 0$, and that $P_{K}(\omega) \equiv 0$ is excluded (as this would mean that $C_{K}(t) \equiv 0$ in contradiction with canonical relation $\left.C_{K}(t=0)=T^{2} d / 2>0\right)$, we find that the integral in eq. C8 is necessarily positive, hence $C_{K}(z) \neq 0$.

In conclusion, we demonstrated that eq. 48 yields a well-defined (unique) and stable solution. 


\section{Appendix D: Some properties of the correlation functions}

Below we list some general relations for the correlation functions obtained with the classical $\mathrm{NH}$ dynamics (tchain $=1$ ) at short times and at low frequencies. These relations come directly from eqs. 25, 26, 29, 30 and the fact that the time-dependent functions are even (due to the time reversibility) and analytical.

Eq. 29 allows to predict $c_{v 0}(t)$ at short $t$ based on $c_{v}(0)=d / 2$ :

$$
c_{v 0}(t)=\frac{d}{2}\left[\frac{\left(\omega_{Q} t\right)^{2}}{2}-\frac{\left(\omega_{Q} t\right)^{4}}{24}+\mathscr{O}(t)^{6}\right], \omega_{Q} t \ll 1
$$

It leads to (at $\left.\omega_{Q} t \ll 1\right)$ :

$$
\begin{gathered}
C_{E}(t)=T^{2}\left[c_{v s}-\frac{d}{4}\left(\omega_{Q} t\right)^{2}+\frac{d}{48}\left(\omega_{Q} t\right)^{4}+\mathscr{O}\left(t^{6}\right)\right], \\
N C_{E T}(t)=T^{2}\left(1-\frac{1}{2}\left(\omega_{Q} t\right)^{2}+\mathscr{O}\left(t^{4}\right)\right), C_{T}(t)=\frac{2 T^{2}}{d}+\mathscr{O}\left(t^{2}\right)
\end{gathered}
$$

Note that the first 3 terms of $C_{E}(t)$ expansion do not reflect any properties of the physical system (but only the thermostat-related frequency $\omega_{Q}$ ).

Turning to $\omega$-dependent functions at low frequencies, $\omega / \omega_{Q} \ll 1$, we get:

$$
\begin{gathered}
C_{E}(\omega)=\mathrm{i} \omega \eta_{E} T^{2}+\mathscr{O}\left(\omega^{2}\right), \quad \eta_{E}=\frac{1}{T^{2}} \int_{0}^{\infty} C_{E}(t) \mathrm{d} t \\
N C_{T E}(\omega)=\frac{2}{d} \frac{\omega^{2}}{\omega_{Q}^{2}} T^{2}\left(-c_{v s}+\mathrm{i} \omega \eta_{E}+\mathscr{O}\left(\omega^{2}\right)\right) \\
C_{T}(\omega)=-\frac{2 T^{2}}{d} \frac{\omega^{2}}{\omega_{Q}^{2}}\left(1+\frac{2}{d} \frac{\omega^{2}}{\omega_{Q}^{2}} c_{v s}+\mathscr{O}\left(\omega^{3}\right)\right) \\
c_{v 0}^{*}(\omega)=c_{v s}-\mathrm{i} \omega \eta_{E}+\mathscr{O}\left(\omega^{2}\right)
\end{gathered}
$$

Noteworthily, the above relations are consistent with the general property of the $\omega$-dependent functions: changing sign of a real $\omega$ results in the complex conjugated function.

In view of eq. 27 the expansion given in the r.h.s. of eq. D7 is also valid for the heat capacity $c_{v}^{*}(\omega)$ :

$$
c_{v}^{*}(\omega)=c_{v s}-\mathrm{i} \omega \eta_{E}+\mathscr{O}\left(\omega^{2}\right)
$$

Using eqs. D4, D5, D6, D7, D8 the constant $\eta_{E}$ can be expressed as

$$
\eta_{E}=\int_{0}^{\infty}\left[c_{v s}-c_{v 0}(t)\right] \mathrm{d} t=\int_{0}^{\infty}\left[c_{v s}-c_{v}(t)\right] \mathrm{d} t=T^{-2} \int_{0}^{\infty} C_{U}(t) \mathrm{d} t
$$


where

$$
C_{U}(t)=\frac{1}{N}\left\langle\delta U\left(t+t^{\prime}\right) \delta U\left(t^{\prime}\right)\right\rangle
$$

is the autocorrelation function of the total potential energy $U$ (cp. eq. 6).

Therefore $\eta_{E}$ must be universal, independent of $Q$. This constant, $\eta_{E}>0$, defines the lowfrequency energy dissipation rate related to a $T$-perturbation. ${ }^{15,34}$

Another interesting feature comes from eq. D6:

$$
\int_{0}^{\infty} C_{T}(t) \mathrm{d} t=0
$$

This relation is exact (no $1 / N$ corrections): it can be derived from the exact equation of $\mathrm{NH}$ dynamics

$$
C_{T}(t)=-\frac{N Q^{2}}{g^{2}} \frac{\partial^{2}}{\partial t^{2}} C_{\xi \xi}(t)
$$

Eq. D11 implies that fluctuations of the time-averaged kinetic energy

$$
\bar{K} \equiv \frac{1}{\Delta t} \int_{0}^{\Delta t} K(t) \mathrm{d} t
$$

are extremely weak if $\Delta t \gg \tau_{\max }$, where $\tau_{\max }$ is the longest relaxation time associated with $C_{T}(t)$. In this case the dispersion of $\bar{K}$ can be approximated as

$$
\operatorname{var}(\bar{K})=\left(\frac{g}{2}\right)^{2} \frac{1}{N} \frac{2}{(\Delta t)^{2}} \int_{0}^{\Delta t} C_{T}(t)(\Delta t-t) \mathrm{d} t \simeq-\left(\frac{g}{2}\right)^{2} \frac{1}{N} \frac{2}{(\Delta t)^{2}} \int_{0}^{\infty} t C_{T}(t) \mathrm{d} t
$$

where the second step uses $\Delta t \gg \tau_{\max }$ and eq. D11. ${ }^{75}$

Using eqs. D12, B7 we get

$$
\int_{0}^{\infty} t C_{T}(t) \mathrm{d} t=-\frac{N T Q}{g^{2}}
$$

and

$$
\operatorname{var}(\bar{K}) \simeq \frac{Q T}{2(\Delta t)^{2}}
$$

It should be compared to the variance of instantaneous $K$ :

$$
\operatorname{var}(K)=\frac{N d}{2} T^{2}
$$

For the system described in Appendix A with $N=10^{4}, \Delta t=10^{5}, Q / T=10 / 3, d=2$ we obtain from eqs. D14, D15:

$$
\frac{\operatorname{var}(\bar{K})}{\operatorname{var}(K)} \simeq \frac{Q}{T}\left(N d(\Delta t)^{2}\right)^{-1}=\frac{5}{3} \cdot 10^{-14}
$$

This result was verified using our simulation data (with sampling time-step $\delta t=0.01$ and MD time-step of 0.005$)$ giving $\operatorname{var}(\bar{K}) / \operatorname{var}(K) \approx 1.60 \cdot 10^{-14}$. 


\section{REFERENCES}

${ }^{1}$ L.D. Landau, E.M. Lifshitz, Statistical Physics (Pergamon Press, Oxford, 1998).

${ }^{2}$ J.P. Hansen and I.R. McDonald, Theory of Simple Liquids (Academic Press, London, 1986).

${ }^{3}$ D. Forster, Hydrodynamic Fluctuations, Broken Symmetry, and Correlation Fluctuations (Benjamin Cummings, London, 1983).

${ }^{4}$ C. Ruscher, A. N. Semenov, J. Baschnagel, and J. Farago, J.Chem.Phys. 146, 144502 (2017).

${ }^{5}$ I. Kriuchevskyi, J. P. Wittmer, H. Meyer, and J. Baschnagel, Phys. Rev. Lett. 119, 147802 (2017).

${ }^{6}$ L. Klochko, J. Baschnagel, J.P. Wittmer, A.N. Semenov, J.Chem.Phys. 151, 054504 (2019)

${ }^{7}$ L. Klochko, J. Baschnagel, J.P. Wittmer, O. Benzerara, C. Ruscher, and A.N. Semenov, Phys.Rev.E 102, 042611 (2020).

${ }^{8}$ J. P. Wittmer, H. Xu, P. Polińska, F. Weysser, and J. Baschnagel, J. Chem. Phys. 138, 191101 (2013).

${ }^{9}$ G. George, L. Klochko, A. N. Semenov J. Baschnagel, and J. P. Wittmer, "Ensemble fluctuations matter for variances of macroscopic variables", European Physical Journal E, 2021.

${ }^{10}$ M. P. Allen and D. J. Tildesley, Computer Simulation of Liquids (Oxford University Press, Oxford, 2017).

${ }^{11}$ R. B. Bird, R. C. Armstrong and O. Hassager, Dynamics of Polymeric Liquids: Fluid Mechanics, Wiley, 1987.

${ }^{12}$ P. Scheidler, W. Kob, A. Latz, J. Horbach, and K. Binder, Phys.Rev. B 63, 104204 (2001).

${ }^{13}$ P.W Anderson, B.I. Halperin, and C.M. Varma, Philos. Mag. 25, 1 (1972).

${ }^{14}$ J.L. Black, Phys. Rev. B 17, 2740 (1978).

${ }^{15}$ N. O. Birge, Phys. Rev. B 34, 1631 (1986).

${ }^{16}$ M.T. Loponen, R.C. Dynes, V. Narayanamurti, and J. P. Garno, Phys. Rev. Lett. 45, 457 (1980).

${ }^{17}$ M.T. Loponen, R.C. Dynes, V. Narayanamurti, and J.P. Garno, Phys. Rev. B 25, 1161 (1982).

${ }^{18}$ M. Meissner and K. Spitzmann, Phys. Rev. Lett. 46, 265 (1981).

${ }^{19}$ Y.-H. Jeong, Progress in experimental techniques for dynamic calorimetry, Thermochimica Acta 304/305, 67 (1997).

${ }^{20}$ W. Götze and A. Latz, J.Phys.:Condens.Matter 1, 4169 (1989).

${ }^{21}$ T. Franosch, M. Fuchs, and A. Latz, Phys.Rev.E 63, 061209 (2001).

${ }^{22}$ E.B. Tadmor and R.E. Miller, Modeling Materials: Continuum, Atomistic and Multiscale Techniques. Cambridge University Press, 2011. 
${ }^{23}$ D.P. Landau, K. Binder, A Guide to Monte Carlo Simulations in Statistical Physics, Cambridge U. Press, Cambridge, 2000.

${ }^{24}$ P.H. Hünenberger, Thermostat Algorithms for Molecular Dynamics Simulations, Adv. Polym. Sci. 173, 105 (2005).

${ }^{25}$ S. Nosé, J. Chem. Phys. 1984, 81, 511; Mol. Phys. 1984, 52, 255.

${ }^{26}$ S. Nosé, Prog. Theor. Phys. Suppl. 103, 1 (1991).

${ }^{27}$ W.G. Hoover, Phys. Rev. A 31, 1695 (1985).

${ }^{28}$ J.K. Nielsen and J.C. Dyre, Phys.Rev. B 54, 15754 (1996).

${ }^{29}$ N. Menon, J. Chem. Phys. 105, 5246 (1996).

${ }^{30}$ S.L. Simon, G.B. McKenna, J. Chem.Phys. 107, 8678 (1997).

${ }^{31}$ L. Mayorga, W.W. van Osdol, J. L. Lacomba, and E. Freire, Proc. Natl. Acad. Sci. USA 85, 9514 (1988).

${ }^{32}$ https://lammps.sandia.gov/doc/Manual.html; S. C. Plimpton, Comput. Phys. 117, 1 (1995).

${ }^{33}$ D. J. Evans and B. L. Holian, J. Chem. Phys. 83, 4069 (1985).

${ }^{34}$ J. K. Nielsen, Phys. Rev. E 60, 471 (1999).

${ }^{35}$ B.L. Holian, A.F. Voter, R. Ravelo, Phys.Rev.E 52, 2338 (1995).

${ }^{36}$ G. S. Grest and S. R. Nagel, J.Phys.Chem. 91, 4916 (1987).

${ }^{37}$ J.L. Lebowitz, J.K. Percus, and L. Verlet, Phys.Rev. 153, 250 (1967).

${ }^{38}$ G.J. Martyna, M.E. Tuckerman and M.L. Klein, J.Chem.Phys. 97, 2635 (1992)

${ }^{39}$ M.E. Tuckerman , Y. Liu, G. Ciccotti and G.J. Martyna, J.Chem.Phys. 115, 1678 (2001).

${ }^{40}$ G.U. Gamboa, J.M. Vásquez-Pérez, P. Calaminici, A.M. Köster, International Journal of Quantum Chemistry 110, 2172 (2010).

${ }^{41}$ C. Bennemann, W. Paul, K. Binder, and B. Dünweg, Phys. Rev. E 57, 843 (1998).

${ }^{42}$ F. Varnik, J. Baschnagel, and K. Binder, Phys. Rev. E 65, 021507 (2002).

${ }^{43}$ D.J. Evans, S. Sarman, Phys.Rev.E 48, 65 (1993).

${ }^{44}$ C.P. Dettmann and G.P. Morriss, Phys.Rev.E 54, 2495 (1996).

${ }^{45}$ D.J. Evans, J. Chem. Phys. 78, 3297 (1983).

${ }^{46}$ W.G. Hoover, A.J.C. Ladd and B. Moran, Phys. Rev. Lett. 48, 1818 (1982).

${ }^{47}$ G.P. Morriss, C.P. Dettmann, Chaos 8, 321 (1998).

${ }^{48}$ A. Tanguy, J. P. Wittmer, F. Léonforte, and J.-L. Barrat, Phys. Rev. B 66, 174205 (2002).

${ }^{49}$ W.G. Hoover, C.G. Hoover, J.Chem.Phys. 126, 164113 (2007).

${ }^{50}$ A. Ninarello, L. Berthier, and D. Coslovich, Phys.Rev.X 7, 021039 (2017). 
${ }^{51}$ F. Wang, D.P. Landau, Phys. Rev. Lett. 86, 2050 (2001); Phys. Rev. E 64, 056101 (2001).

${ }^{52}$ M.P. Taylor, W. Paul, K. Binder, J.Chem.Phys. 131, 114907 (2009).

${ }^{53}$ K. Binder, J.Non-Cryst.Solids 274, 332 (2000).

${ }^{54}$ K. Binder, Monte Carlo and Molecular Dynamics Simulations in Polymer Science, Oxford, NY, 1995.

${ }^{55}$ M. D’Alessandro, A. Tenenbaum, A. Amadei, J. Phys. Chem. B 106, 5050 (2002).

${ }^{56}$ J.R.Brown, J.D.McCoy, D.B.Adolf, J.Chem.Phys. 131, 104507 (2009).

${ }^{57}$ Here and below $T$ is given in energy units, and the Boltzmann constant is set to 1 .

${ }^{58}$ Strictly speaking, here $\omega$ should be considered as a complex number with infinitesimal and negative imaginary part, $\mathfrak{I}(\omega) \rightarrow 0$.

${ }^{59}$ Note that $c_{v 0}(t)$ becomes weakly time-dependent for $t>200$ below the glass transition, at $T<$ 0.26 .

${ }^{60}$ Here and below we assume that $g=d N$, where $d$ is the space dimension and $N$ is the number of classical particles in the system. In MD simulation studies the centre-of-mass velocity is often set to 0 leading to $g=d(N-1)$. However, the difference between $N$ and $N-1$ is normally totally negligible for large systems used in modern simulations.

${ }^{61}$ Note that in simulations $T_{\mathrm{i}}(t)$ can precisely follow eq. 9 with no fluctuations on top of it.

${ }^{62}$ Note that a heat can never be injected directly to configurational degrees of freedom.

${ }^{63}$ Note that $C_{E T}(\omega)=C_{T E}(\omega)$ due to time reversibility.

${ }^{64}$ Here and below we omit small corrections due to the $\xi^{2}$-term in eq. 22 . A simple analysis shows that the corresponding corrections are of the relative order $\mathscr{O}(1 / N)$ for equilibrium correlation functions considered below. Trying to obtain these corrections is a rather pointless task since any intensive thermodynamic quantity of a finite system deviates anyway by $\mathscr{O}(1 / N)$ from its thermodynamic limit. This effect is due to an incorrect spectrum of long-wave fluctuation modes for a finite system as compared to its infinite counterpart.

${ }^{65}$ Note that the canonical $C_{T}(\omega)$ is related to the microcanonical correlation function of temperature, $C_{T}^{(m c)}(\omega)$, via a Lebowitz transformation ${ }^{37}: C_{T}(\omega)=\frac{T^{2}}{c_{v s}}+C_{T}^{(m c)}(\omega)$ (cf. eq. (21) in ref. ${ }^{12}$ ). ${ }^{66}$ The precise way to obtain $c_{v}(t)$ based on correlation functions is described in the next section. For the model system we consider the resultant $c_{v}(t)$ indeed does not show any oscillations (cf. Fig. 5) in spite of strongly oscillating $C_{T}(t)$.

${ }^{67} C_{T}(z)$ cannot have singularities at $\mathfrak{I}(z)<0$ since $C_{T}(t)$ must decay at long $t$. Moreover, $\mathfrak{I}(z)=0$ is also practically impossible since in this case eq. 33 would imply $z \neq 0$ and $\mathfrak{I}\left(c_{v}^{*}(z)\right)=0$ which 
means no dissipation at the frequency $z$, cf. eq. C4.

${ }^{68}$ Of course the system must be first equilibrated at $\omega_{Q} \sim \omega_{Q}^{*}$ since velocity equilibration at very high $\omega_{Q}$ (with $\mathrm{NH}$ dynamics for tchain $=1$ ) may be totally inefficient.

${ }^{69}$ To prove this let's assume the opposite: $\varphi\left(\omega_{Q}\right) \neq 0$. In this case the second eq. 40 ensures that $\varphi\left(\omega_{Q}\right)+\psi\left(\omega_{Q}\right)=0$, which gives $c_{v}^{*}\left(\omega_{Q}\right)=d / 2$ on recalling eq. 39. Finally, using eq. 29 we arrive at $c_{\nu 0}^{*}\left(\omega_{Q}\right)=\infty$ and $C_{E}\left(\omega_{Q}\right)=\infty$, which is physically impossible (as $C_{E}(t)$ must exponentially decrease at long enough $t$ ). We thus get a contradiction implying that $\varphi\left(\omega_{Q}\right)=0$.

${ }^{70}$ For simplicity here we also assume that the system dynamics are time-reversible.

${ }^{71}$ These observations indicate that $C_{U K}(t) / T^{2}$ is really small for $t>1$.

${ }^{72}$ An additional clarification may be useful here: some canonical thermostats (like NH thermostat) involve auxiliary variables ( $\xi$ in the $\mathrm{NH}$ case) and the corresponding coupling parameters (like thermal mass $Q$ ). The basic equation 11 is valid provided that the perturbation of the imposed temperature $T_{\mathrm{i}}$ is supplemented by such adjustment of the coupling parameters that conserves the equilibrium distribution of the thermostat variables. However, this formal requirement (which reduces to setting $Q / T_{\mathrm{i}}=$ const in the $\mathrm{NH}$ case) is not really important. Eqs. 11, 13 remain valid in the thermodynamic limit even without the $Q$-adjustment: for a finite $N$ the relative error still scales as $1 / N$.

${ }^{73}$ More precisely, $\delta v / v \sim \xi / \omega_{Q}$ for $\omega_{Q} \tau \gtrsim 1$, and $\delta v / v \ll \xi / \omega_{Q}$ for $\omega_{Q} \tau \ll 1$.

${ }^{74}$ Following a reviewer's request we introduced a random noise in the $C_{U K}(t)$ correlation function and found that a noise of relative amplitude $\sim 5 \%$ did not lead to any detrimental effect for $c_{v}(t)$ : the precision of final data remained virtually the same $\left(\sim 3 \cdot 10^{-4}\right)$. In practice, the noise in $C_{U K}(t)$ is weaker, whereas $C_{U K}(t)$ itself is also much smaller than $C_{E}(t)$ (see also note ${ }^{71}$ and the beginning of sect. VI A).

${ }^{75}$ Note that here we consider variations of kinetic energy across the ensemble, rather than along the trajectory, and that is why the integrand in the above equation is proportional to $C_{T}(t)$, and not to the difference $C_{T}(0)-C_{T}(t)$. 


\section{FIGURE CAPTIONS}

FIG. 1. The approximate time-dependent (transient) heat capacity, $c_{v 0}(t)$, eq. 5 , for the $2 \mathrm{D}$ pLJ model (of Appendix A) obtained using the NH thermostat with tchain $=1$ and different thermal mass parameters $Q: Q=4 / 3$ (blue), 40 (black), 400 (red), 8000 (brown). Temperature $T=0.4$; $t$ is time in LJ units.

FIG. 2. (a) The dependence of the relaxation rate $\gamma^{*}$ of the temperature autocorrelation function $C_{T}(t)$ on the Nosé frequency $\omega_{Q}$ for the model of eq. 37 with $\alpha=1$ and $\kappa=2$ (solid line), $\kappa=5$ (dotted line). The maxima are indicated with crosses. (b) The transient heat capacity $c_{v}(t)$ for the same model. The solid and dotted curves correspond to $\kappa=2$ and 5 , respectively; the vertical dashed segments indicate $t_{1 / 2}$. Note that the shape of all the curves does not depend on $\alpha$.

FIG. 3. Time-dependent correlation functions obtained based on NH simulations of the $2 \mathrm{D}$ pLJ system at $T=0.4$, total sampling time $\Delta t=10^{5}$ (in LJ units): $C_{U}(t) / T^{2}$ (solid curve), $C_{K}(t) / T^{2}$ (dashed curve), $C_{E}(t) / T^{2}$ (dots) for (a) tchain $=1, Q=4 / 3$; (b) tchain $=1, Q=400$; (c) tchain $=3, Q=4 / 3$.

FIG. 4. The time-dependent heat capacity $c_{v}(t)$ calculated based on the correlation function $C_{U}(t)$ for $T=0.4$, tchain=1, $Q=4 / 3$ (black curve), 40 (red), 400 (blue), 8000 (brown). The sampling time step $\delta t=0.01$ for $Q=4 / 3 ; \delta t=0.05$ otherwise.

FIG. 5. The transient heat capacity $c_{v}(t)$ in semilog scale for $T=0.4, Q=4 / 3$ obtained based on $\mathrm{NH}($ tchain $=1)$ and $\mathrm{NH}$-chain (tchain = 3) simulations. The 2 curves coincide to the precision of the data. The horizontal line indicates the static heat capacity $c_{v s} \approx 1.9431$. 


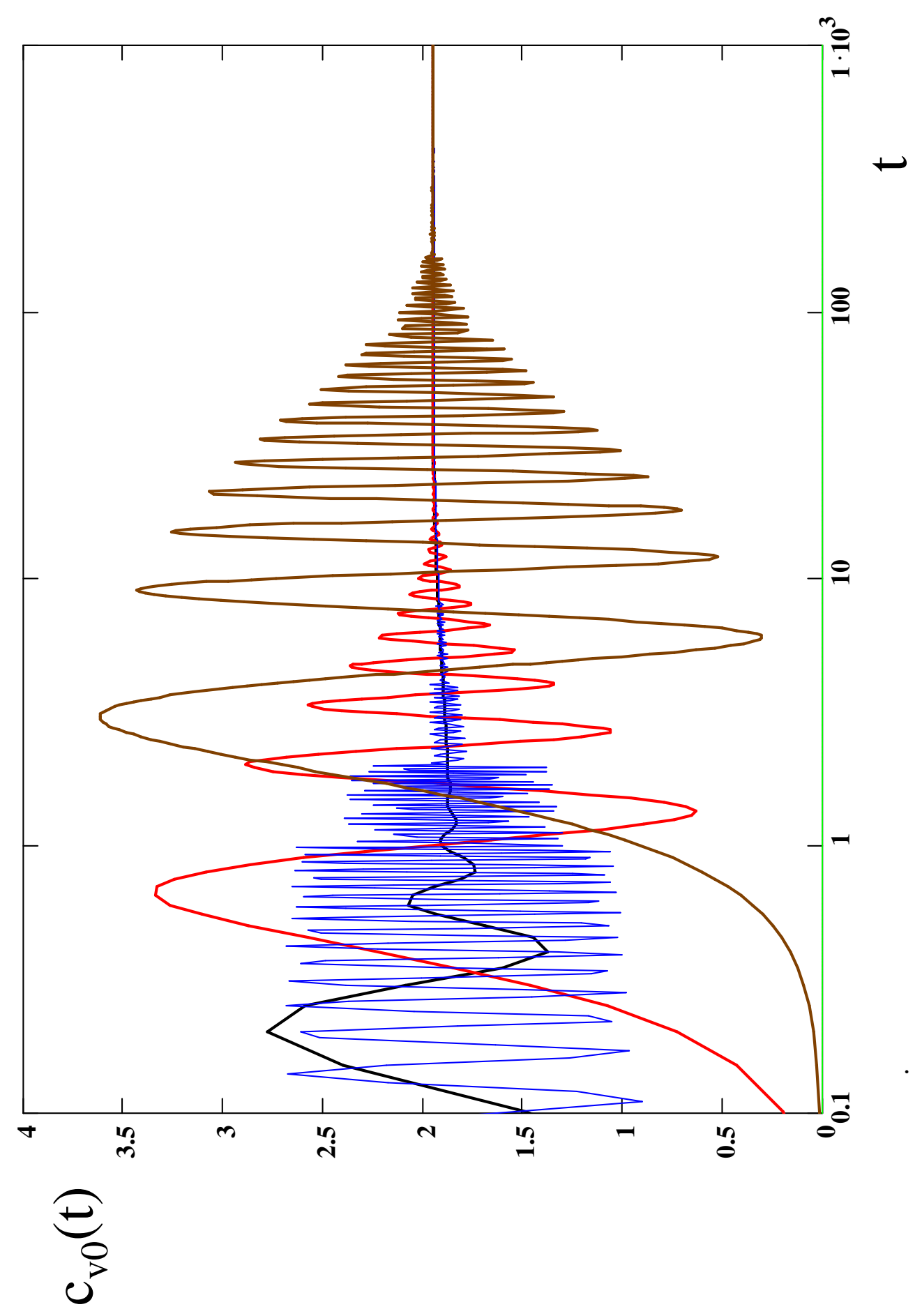

$\underset{0}{\infty}$ 

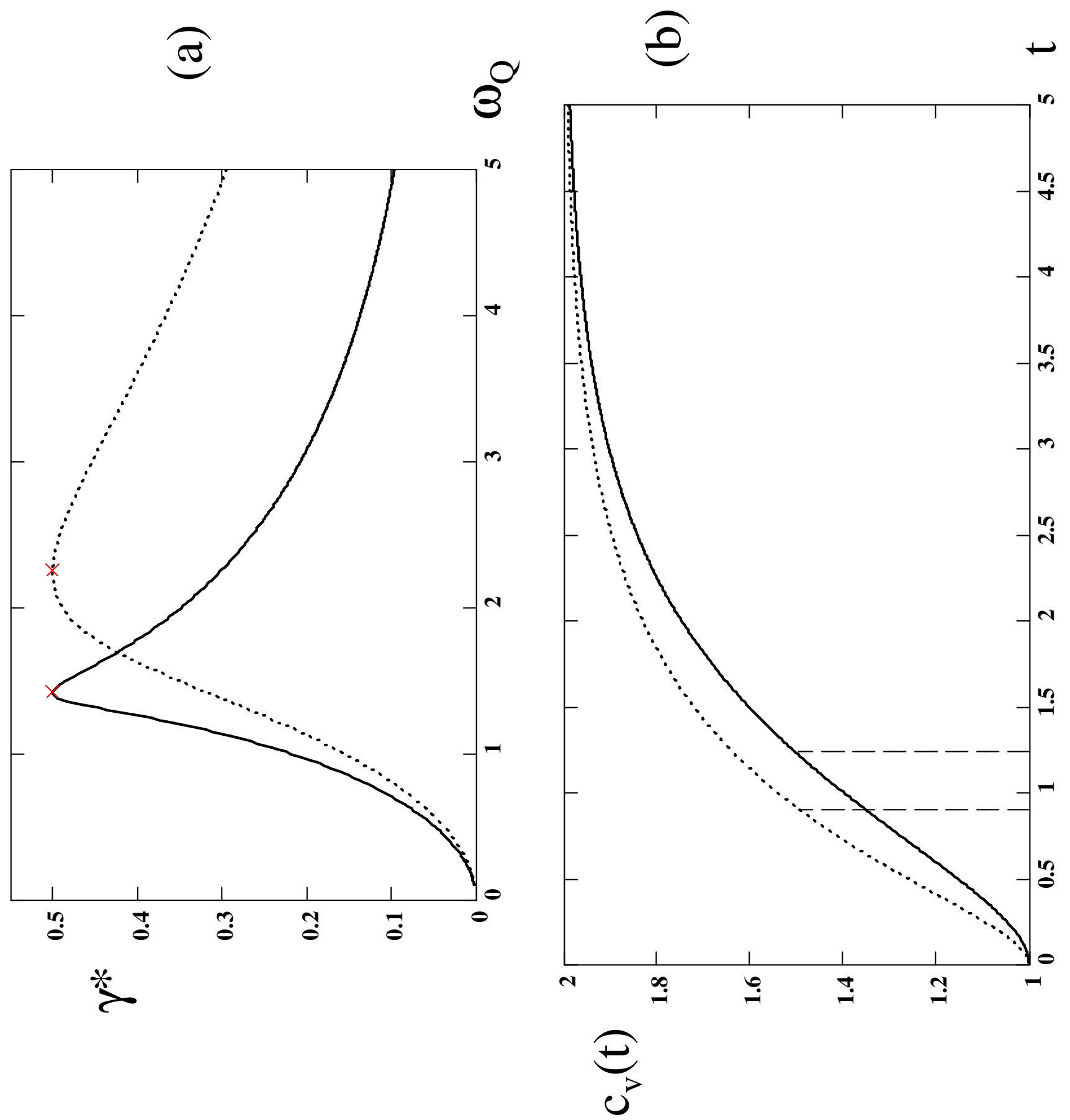

$N$

is 

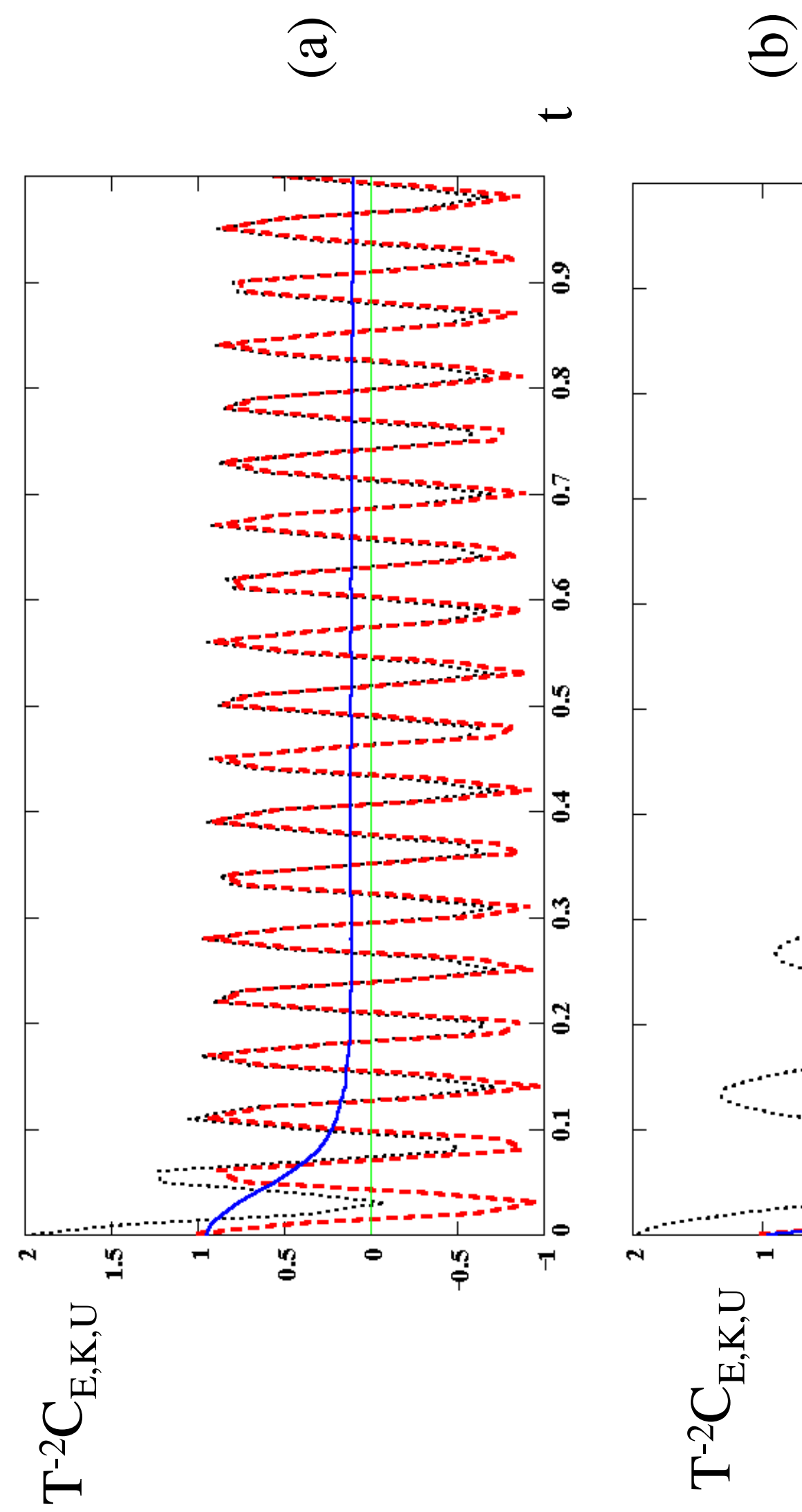


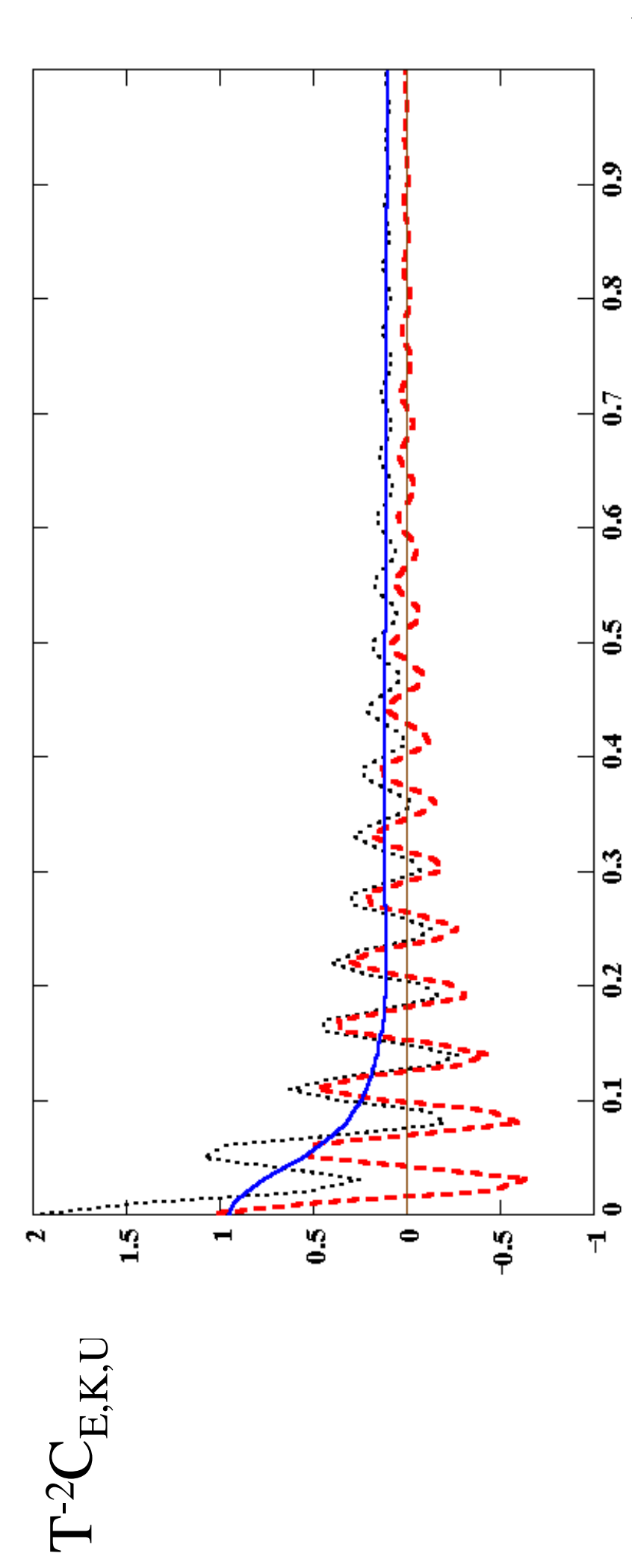

m 

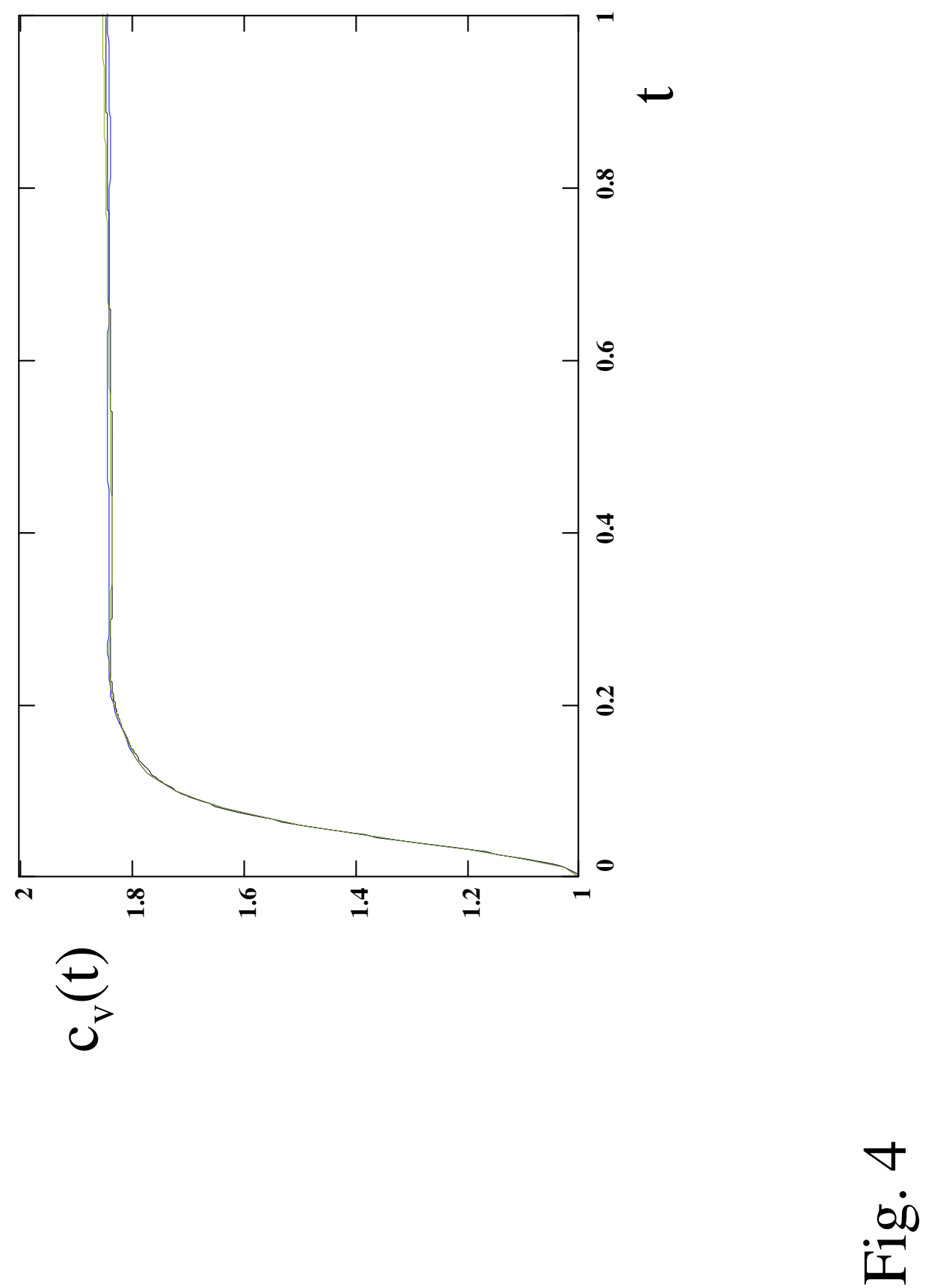


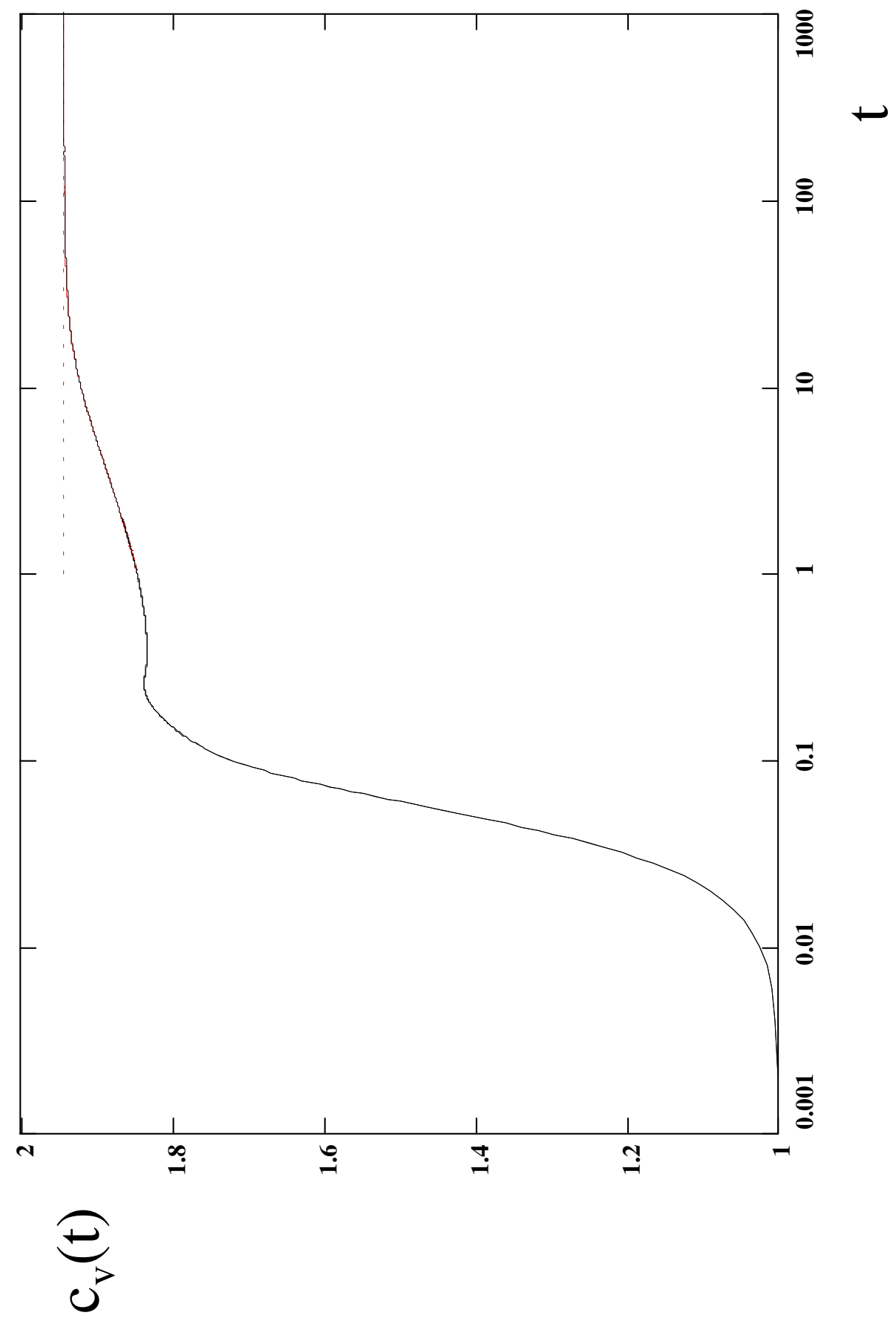

n 